\title{
Jenseits des methodologischen Nationalismus
}

\section{Außereuropäische und europäische Variationen der Zweiten Moderne1}

\author{
Von Ulrich Beck und Edgar Grande
}

Zusammenfassung: Die Kernfragen dieses einleitenden Aufsatzes lauten: Wie können Gesellschafts- und Politiktheorie theoretisch, methodologisch und empirisch für eine historisch neue, „verflochtene“ Moderne geöffnet werden, die ihre eigenen Grundlagen aufhebt? Wie kann die Theorie die fundamentale Zerbrechlichkeit und Wandelbarkeit der gesellschaftlichen Dynamiken (unbeabsichtigte Nebenfolgen, Herrschaft und Macht) erschließen, die am Beginn des 21. Jahrhunderts von der Globalisierung von Kapital und Risiken ausgehen? Was meint „methodologischer Nationalismus", welche theoretischen und methodologischen Probleme entstehen hier, und wie kann die theoretische und empirische Forschung sich für diese öffnen? Im Folgenden entwickeln wir diese "kosmopolitische Wende" in der soziologischen und politischen Theorie und Forschung in vier Schritten: Zunächst präsentieren wir die wichtigsten begrifflichen Werkzeuge einer Theorie der kosmopolitischen Modernen; danach de-konstruieren und re-definieren wir das Modell westlicher Modernisierung anhand von Beispielen aus der Forschung über Individualisierung und Risiko; drittens greifen wir das Schlüsselproblem des methodologischen Kosmopolitismus auf, nämlich die Definition der angemessenen Untersuchungseinheit; und schließlich diskutieren wir, viertens, Perspektiven und Dilemmata einer Theorie kosmopolitischer Modernen, insbesondere Probleme des Adressatenbezugs und des politischen Handelns.

\section{Einleitung: Die kosmopolitische Wende in der Gesellschafts- und Politiktheorie}

Wenn eine Weltordnung zusammenbricht, beginnt das Nachdenken darüber. Das gilt nicht für den heute vorherrschenden Typus der Gesellschaftstheorie, der in universalistischer Erhabenheit und schlafwandlerischer Sicherheit über den Niederungen des epochalen Wandels (Klimawandel, Finanzkrise, Krise der Demokratie und der nationalstaatlichen Institutionen) hinweg schwebt. Diese universalistische Gesellschaftstheorie, sei es nun eine strukturalistische, interaktionistische, marxistische, Kritische oder Systemtheorie, ist heute antiquiert und provinziell. Antiquiert ist sie, weil sie ausschließt, was zu beobachten ist: ein Paradigmenwechsel von Gesellschaft und Politik in der Moderne (von der Ersten zur Zweiten Moderne). Provinziell ist sie, weil sie den pfadabhängigen Erfahrungs- und Erwartungsraum der westeuropäischen oder auch der US-amerikanischen Modernisierung fälschlich verabsolutiert und damit gerade den soziologischen Blick auf deren Besonderheit verstellt. Man würde bei weitem zu kurz greifen, wenn man meinte, dass wir, die europäische Soziologie, die Modernisierungspfade der Anderen nur ergänzend verstehen müssen, weil sonst unser Weltbild unvollständig ist. Vielmehr gilt: wir Europäer können uns nur dann selbst verstehen, wenn wir uns ,deprovinzialisieren“, das heißt, soziologisch-methodologisch mit den Augen der Anderen sehen lernen.

Hauptgegenstand dieses Schwerpunktheftes der Sozialen Welt ist daher die Notwendigkeit einer kosmopolitischen Wende in der soziologischen und politischer Theorie und Forschung. Die Kernfrage dieses einleitenden Aufsatzes lautet: Wie können Gesellschafts- und Politiktheorie theoretisch, methodologisch und empirisch für eine historisch neue, ,verflochtene“

1 Dieser Text (sowie das Konzept dieses Schwerpunkt-Heftes) entstanden im Rahmen des Sonderforschungsbereiches 536 ,Reflexive Modernisierung“ der Universität München. Wir danken den Kolleginnen und Kollegen dieses Forschungszusammenhangs für anregende Diskussionen und der DFG für großzügige Finanzierung. 
Moderne am Beginn des 21. Jahrhunderts geöffnet werden, die ihre eigenen Grundlagen aufhebt? Wie kann die Theorie die fundamentale Zerbrechlichkeit und Wandelbarkeit der gesellschaftlichen Dynamiken (unbeabsichtigte Nebenfolgen, Herrschaft und Macht) erschließen, die von der Globalisierung von Kapital und Risiken ausgehen? Welche theoretischen und methodologischen Probleme entstehen hier, und wie kann die empirische Forschung sich für diese öffnen, sich ihnen widmen?

Wenn es um die Globalisierung von Kapital und Risiko geht, ist es mittlerweile eine Binsenweisheit, dass nationale Institutionen alleine die Regulierung des globalen Kapitalismus und die neuen globalen Risiken nicht bewältigen können (Beck 2007). Zugleich ist aber auch kein „Weltstaat" vorhanden und keine internationale Organisation in der Lage, Kapital und Risiko auf globaler Ebene in auch nur annähernd gleicher Weise zu regulieren wie der „Keynesianische Wohlfahrts-Nationalstaat" (Jessop 2002) innerhalb der Ersten, industriellen Moderne. Statt dessen beobachten wir eine komplexe Rekonstituierung politischer Herrschaft jenseits des Nationalstaats, die von neuen Formen politischer Interdependenz geprägt ist und die derzeit die ökonomische Regulierung sowie das Management und die Kontrolle von Risiken auf globaler Ebene bestimmen (Grande / Pauly 2005). Die „Weltrisikogesellschaft” (Beck 1999, 2007) ist heute ein außerordentlich schwieriges Terrain, das sich unter anderem aus koordinierten nationalen Politiken, bilateralen und multilateralen Abkommen, inter-, trans- und supranationalen Institutionen, transnationalen Konzernen, privaten Stiftungen und Netzwerken der Zivilgesellschaft konstituiert. Trotz dieser rapide anwachsenden Zahl globaler Organisationen und transnationaler Institutionen ist jedoch ein zunehmendes Unbehagen zu verzeichnen, das sich nicht zuletzt aus den zögerlichen Reaktionen auf die Weltfinanzkrise, den drohenden Staatsbankrott, die Gefährdung des Euro (und damit vielleicht sogar der EU), aber auch die enttäuschenden Ergebnisse der Klimakonferenz am Ende des Jahres 2009 in Kopenhagen nährt; ein Unbehagen darüber, dass diese Institutionen sich als unfähig erweisen, die Herausforderungen zu bestehen, derentwegen sie geschaffen wurden. Ähnliche Entwicklungen und Enttäuschungen sind auf nationaler Ebene zu beobachten, was die demokratischen Institutionen und die sozialen Sicherungssysteme, aber auch etwa die Institution der Familie betrifft.

Kann die Weltbank das globale Armutsproblem lösen? Kann die Ernährungs- und Landwirtschaftsorganisation der Vereinten Nationen (FAO) eine globale Ernährungskrise bewältigen? Kann die Welthandelsorganisation (WTO) wirksam den globalen Handel regulieren? Es scheint, als böten diese Institutionen keine ausreichende Basis zur Bewältigung oder auch nur Kontrolle der globalen Risiken und Krisen, die letztlich aus dem globalen Sieg des Industriekapitalismus hervorgehen. Genau dies ist es, was die transformative Dynamik der Zweiten, kosmopolitischen Moderne ausmacht. Klafft denn kein Bruch der Jahrhunderte zwischen den Gefahren, Chancen und Konfliktdynamiken der grenzüberschreitenden, radikalisierten Modernisierung des 21. Jahrhunderts und den noch im 19. Jahrhundert wurzelnden Kategorien, Ideen, Institutionen und Strukturen des Industriekapitalismus und der nationalstaatlichen Herrschaftsform?

Und weiter: Was bedeutet all dies für die Konfliktdynamiken, durch die angesichts national und global wachsender sozialer Ungleichheiten Fragen der sozialen Gerechtigkeit aufgewirbelt und im Ringen um die Schaffung transnationaler Regime und Institutionen allgegenwärtig werden? Wo und wie kommen jenseits der schnellen Lippenbekenntnisse praxiswirksam kosmopolitische Konstellationen, Handlungsweisen und Aktivitäten zustande? Entstehen sie eher im Zentrum oder in der Peripherie? Eher oben oder unten? Eher erzwungen oder freiwillig? Können wir annehmen, dass die Vielfalt der Pfade und Abhängigkeits- bzw. Interdependenzkonstellationen der Zweiten Moderne dem integrativen Druck widerstehen werden, der von einer sich globalisierenden Wirtschaft, der globalen Ausbreitung von Menschenrechten oder dem „kosmopolitischen Imperativ” der Weltrisikogesellschaft - „kooperiert oder scheitert!”- 
ausgeht? Wie können ,imaginierte kosmopolitische Risikogemeinschaften” über territoriale Grenzen und soziale Spaltungen hinweg geschaffen werden, die neue Möglichkeiten der Verständigung und des Handelns eröffnen, ja erfordern?

In diesem Einleitungsaufsatz wollen wir einige der konzeptionellen Werkzeuge vorstellen, die benötigt werden, um solche Fragen zu beantworten. Wir argumentieren, dass man nicht sinnvoll über „methodologischen Kosmopolitismus“ sprechen kann, ohne die Mauern des Eurozentrismus einzureißen. Wir müssen in Europa den Blick für die Welten jenseits Europas öffnen, für die globalen Verflechtungen der Geschichten von Kolonialisierung und Beherrschung und für die grenzüberschreitenden Dynamiken, Dependenzen, Interdependenzen und Vermischungen. Wie? Durch eine neue Begriffsarchitektur, die zwei Typen von Gesellschaftstheorie unterscheidet: die singulare und die plurale; eine Theorie der Gesellschaft im Singular, die weder national noch global ist, sondern absolut in universalistischen Begriffen verstanden wird, von einer Theorie von Gesellschaften im Plural, die sich auf sehr verschiedene Pfade und Kontexte von Modernisierungsprozessen bezieht.

Die soziologische Theorie war von Anfang an durch das Bemühen geprägt, eine allgemeine Theorie der (modernen) Gesellschaft im Singular zu formulieren, also universelle Begriffe, Prinzipien, Strukturen, Systeme und Arten des sozialen Handelns und Wandels zu identifizieren. Dies ist nicht mehr hinreichend, wenn es das je war. Es (ver)führt im Gegenteil zu dem Kategorienfehler, von den am Beispiel einer Gesellschaft gewonnenen Hypothesen und Einsichten unreflektiert auf die Gesellschaft, das soziale Handeln (im Allgemeinen) zu schließen, die daraufhin (ihrem Anspruch nach) zum universellen Bezugsrahmen verabsolutiert wird. Genau das ist in den meisten vorherrschenden Theorien zeitgenössischer Soziologie der Fall (Bourdieu, Coleman, Foucault, Giddens, Goffman, Habermas, Luhmann, Meyer, Parsons, auch Becks „Risikogesellschaft”). Diese Verwechslung einer Theorie einer Gesellschaft (von vielen) mit der Theorie der Gesellschaft bezeichnen wir als Selbstprovinzialisierung der Gesellschaftstheorie. Die Form der Abstraktion, die für diese Art von Theorie charakteristisch ist, zeugt nicht von professioneller Raffinesse, sondern von einem Mangel an Selbstreflexion sowie der Unfähigkeit, die Wandlungsdynamik moderner Gesellschaften im 21. Jahrhundert zu begreifen.

Die historische und kulturelle Besonderheit zeitgenössischer Gesellschaftstheorie, ja, ihr falscher Universalismus zeigt sich an zwei hervorstechenden Merkmalen. Das erste ist ihr methodologischer Nationalismus (Beck 1997, 2004; Wimmer / Glick Schiller 2002; Weiß 2010): Lange Zeit unterstellte die Gesellschaftstheorie als ihre Untersuchungseinheit den Nationalstaat; die Begriffe „Gesellschaft” und „Kultur” bezogen sich unreflektiert auf das, was man als abgegrenzte, unabhängige und relativ homogene Einheiten wahrnahm, die sich durch nationale Grenzen, Institutionen und Gesetze konstituierten. Dementsprechend ging das theoretische Nachdenken von der unhinterfragten Annahme aus, dass sich Nation, Territorium, Gesellschaft und Kultur nahtlos ineinander fügen. Zweitens wurden, was sich in den Geistesund Sozialwissenschaften ganz allgemein zeigt, viele Grundlagen der Gesellschafts- und Politiktheorien im Zeitalter europäischer Imperien gelegt. Die politische Herrschaft über und die ökonomische Aneignung nicht-westlicher Territorien, Ressourcen und Bevölkerungen bildete den Hintergrund, vor dem westliche Autoren sich mit anderen Gesellschaften befassten. Hierdurch wurden immer wieder abwertende Haltungen gegenüber den kolonisierten Gesellschaften gleichsam analytisch versteift, die man als ,traditional“, das heißt, minderwertig und rückständig im Vergleich zu ihren europäischen und amerikanischen Gegenstücken ansah. Viele Theorien der Moderne beruhen daher auf einem sehr begrenzten Spektrum frühmoderner, nationaler Erfahrungen (die z.B. England in der Ökonomie gesammelt hat, Frankreich in der Politik und Preußen-Deutschland in der Bürokratie), denen man eine universelle Gültigkeit zuschreibt, zumindest aber eine Vorbildfunktion für andere Weltregionen. 
Wir behaupten in diesem Aufsatz nicht, dass eine universelle Theorie ,der” modernen Gesellschaft grundsätzlich unmöglich sei. Wir kritisieren jedoch den hegemonialen Kurzschluss von einer Nationalgesellschaft auf die Gesellschaft im Allgemeinen. In der Zweiten Moderne muss die soziologische und politikwissenschaftliche Theoriebildung die ganze „Pluralität” der Modernisierungspfade durchschreiten, die westlichen und nicht-westlichen Erfahrungen und Perspektiven mitsamt ihren Dependenzen, Interdependenzen und Interaktionen einbeziehen. Dies ist es im Kern, was wir „methodologischen Kosmopolitismus“ (Beck 2002, 2004; Beck / Grande 2004; Beck / Sznaider 2006 b) nennen: einen Ansatz, der die Variationen der Moderne und ihre globalen Interdependenzen als Ausgangspunkt theoretischer Überlegungen und empirischer Forschung nimmt. Im Folgenden entwickeln wir diese „,kosmopolitische Wende” der Gesellschafts- und Politiktheorie in vier Schritten: Zunächst präsentieren wir die wichtigsten begrifflichen Werkzeuge einer Theorie der kosmopolitischen Modernen; danach de-konstruieren und re-definieren wir das Modell westlicher Modernisierung anhand von Beispielen aus der Forschung über Individualisierung und Risiko; drittens greifen wir das Schlüsselproblem des methodologischen Kosmopolitismus auf, nämlich die Definition der angemessenen Untersuchungseinheit jenseits von nationaler Gesellschaft und Nationalstaat; und schließlich diskutieren wir, viertens, normative Probleme und Dilemmata einer Theorie kosmopolitischer Modernen, insbesondere Probleme des Adressatenbezugs und des politischen Handelns.

\section{Theorie kosmopolitischer Modernen: Theoretische Werkzeuge}

Eine der Grundprämissen der klassischen Modernisierungstheorie besteht in dem faszinierenden Gedanken einer Art Diffusion oder eines Transfers von europäischen Theorien in die Peripherie. Alle Gesellschaften würden langfristig die für die Moderne klassische Unterscheidung von Tradition und Moderne preisgeben und die für die westliche Moderne typischen institutionellen Muster hervorbringen, die ,,als weltweites Repertoire“ (Stichweh 2000: 256) verfügbar sind. Zwar seien die verschiedenen Länder und Regionen der Welt in unterschiedlichem Maße entwickelt, ,aber wenn ein Land oder eine Region dem Pfad der Modernisierung folgt, strebt es (sie) mehr oder weniger dem gleichen Ziel zu. [...] Alle nichtmodernen Kulturen und Sozialstrukturen müssen letzen Endes modernen weichen. Dies ist ihr unvermeidliches Schicksal“ (Berger 2006: 202ff). Es gäbe zwar Varianten der Moderne, aber keine Pluralität im Sinne von Modernen mit verschiedenen Kriterien für Modernisierung. Überall werde das „gleiche Stück mit unterschiedlichen Rollen gespielt“ (Berger 2006: 203). Als Konsequenz dieser Diffusionsprozesse stellt die empirische Sozialforschung fest, dass wir in einer Welt leben, die immer mehr zusammenwächst. Es scheint ausgemacht, dass die Gesellschaft im 21. Jahrhundert als „Weltgesellschaft“, das heißt: in einer bestimmten Hinsicht als „,eine“ Entität wahrgenommen wird, über die sich eine Art „Metakultur“ (Stichweh 2000: 22; Meyer 2005) spannt. In letzter Konsequenz würde dies bedeuten, dass mit dem Abschluss des Universalprojekts der Modernisierung das „Ende der Geschichte” (Fukuyama 1992) erreicht wäre.

Diese Erwartung von Konvergenz - eines homogenen und universellen Modells von (westlicher) Modernität, dem man früher oder später überall folgen werde-, ist das genaue Gegenteil unserer Theorie kosmopolitischer Modernen. Das Ziel einer „kosmopolitischen Wende” in der soziologische Theorie besteht darin, diese für die Möglichkeit einer Vielgestaltigkeit verschiedener und autonomer, miteinander verknüpften Modernen (eine „Pluralität der Modernen”), aber auch für neue, globale Imperative und Zwänge zu öffnen. Diese neuen ,kosmopolitischen Imperative"2 sind nicht universalistisch vorgegeben, sondern bilden sich aus der „Interferenz der Nebenfolgen“ (Beck 2002: 179ff) zu Beginn des 21. Jahrhunderts heraus und schaffen beides: neue Konfliktdynamiken sowie neue Prozesse der Risikovergemeinschaftung.

2 Zum Beispiel das Menschenrechtsregime (Levy / Sznaider 2010) oder in Bezug auf globale Risiken der kosmopolitische Imperativ „Kooperieren oder scheitern!“ (siehe später). 
Beide Annahmen müssen begründet werden, und in diesem Abschnitt wollen wir die wichtigsten Bausteine einer Theorie kosmopolitischer Modernen vorstellen. Die Schlüsselfrage, von der wir ausgehen, lautet: Wie können verschiedene Arten moderner Gesellschaften überhaupt theoretisch-konzeptionell erfasst werden? Wie lassen sich die Annahmen der Universalität und Homogenität überwinden, die in konventionellen Gesellschafts- und Systemtheorien vorherrschen? Was ist mit „Pluralität moderner Gesellschaften” und „Modernisierungspfaden" gemeint? Woher kommt diese Pluralität und was folgt aus ihr? Zur Beantwortung dieser Fragen bedienen wir uns dreier theoretischer Denkfiguren, die wir systematisch miteinander verbinden: (1) Pluralität der Modernen und ihre Verzahnung; (2) die Unterscheidung von Erster und Zweiter Moderne sowie (3) Kosmopolitisierung: der Prozess, durch den sich nationale Gesellschaften gegenseitig verinnerlichen.

\section{Pluralität der Modernen und ihre Verzahnung}

Die erste Denkfigur kann in kritischem Austausch mit der Idee multipler Modernen eingeführt werden, wie sie insbesondere von Shmuel N. Eisenstadt (2000 a,b, 2006) und Göran Therborn (1995) entwickelt wurde. ${ }^{3}$ Nach Eisenstadt sind moderne Gesellschaften von strukturellen Widersprüchen geprägt, die auf verschiedene Weise politisch artikuliert und sozial institutionalisiert werden können. Der historische Vergleich zeigt, dass die ,westliche” Moderne keinen universellen Typ sozialer Modernisierung darstellt, sondern nur eine spezielle Kombination jener Institutionen, durch die diese strukturellen Antinomien reguliert werden. Außerhalb Europas, zum Beispiel in China und Japan, findet man andere Formen von Moderne, die - und das ist entscheidend - nicht einfach als strukturelle Variationen der europäischen Moderne aufgefasst werden können, sondern unabhängige, eigenständige Typen von Gesellschaft darstellen.

Im Zusammenhang einer Theorie kosmopolitischer Modernen muss die Idee ,multipler Modernen" allerdings in zwei Richtungen weiterentwickelt werden. In Eisenstadts Theorie sind die Mechanismen und Prozesse, die den strukturellen Unterschieden der einzelnen Typen zugrunde liegen, sowie die Beziehungen dieser Typen zueinander unzureichend ausgearbeitet. Die jeweiligen Typen werden als relativ geschlossene Einheiten aufgefasst, deren Herausbildung als Produkt interner Mechanismen und Prozesse erscheint. Keines von beiden kann aber verallgemeinert werden. Hier setzt Göran Therborn (1995) an und argumentiert, die Unterschiede zwischen modernen Gesellschaften seien Produkte verschiedener Pfade, ,in und durch die Moderne". In der postkolonialen Theorie (Gilroy 1993; Conrad / Randeria 2002; Young 2001) sowie in der „Globalen” bzw. „Atlantischen Geschichte” (Bayly 2006; Benjamin 2009; Osterhammel 2009) wird dieser Aspekt systematisch vertieft. Diese Studien legen überzeugend dar, dass die verschiedenen gesellschaftlichen Modernisierungsprozesse von Anfang an miteinander verzahnt und die einzelnen Gesellschaften in komplexe Abhängigkeiten verwickelt waren. Das gilt auch für die ,westliche”, europäische Moderne. Konzept und Geschichte der „Atlantischen Welt” bieten eine hervorragende Illustration dieser Sichtweise. Das Hauptaugenmerk liegt hier auf den „Verbindungen, Interaktionen und Austauschprozessen, die sich seit dem fünfzehnten Jahrhundert quer über den Atlantik vollziehen. Diese Beziehungen und Interaktionen haben europäische, westafrikanische und indigene amerikanische Gesellschaften transformiert und neue Völker, Gesellschaften, Kulturen, Ökonomien und Ideen an allen Ufern des Atlantik hervorgebracht" (Benjamin 2009: xxiii).

3 Eine kritische Diskussion dieses Theoriestranges findet man in Arnason 2003; vergleichende Fallstudien, die auf Eisenstadts Theorie aufbauen, u.a. in Schwinn 2006. 


\section{Diskontinuierlicher Wandel der Moderne: Die Unterscheidung zwischen Erster und Zweiter Moderne}

Die Idee multipler Modernen und ihrer Verflechtungen bedarf jedoch der Ausweitung in noch einer zweiten Richtung: die Möglichkeit diskontinuierlicher Transformationen innerhalb der Moderne. In den vergangenen zwanzig Jahren wurde dieser Gedanke vor allem im Rahmen der Theorie reflexiver Modernisierung entwickelt (Beck 1986, 1993, 2007 Kapitel XII; Beck / Giddens / Lash 1996; Beck / Bonß / Lau 2001; Beck / Lau 2005; Giddens 1991, 1996, 1997). Diese Theorie beruht auf der Unterscheidung zwischen Grundprinzipien und Basisinstitutionen der Moderne, einer Unterscheidung, die die theoretische Möglichkeit eines strukturellen Bruches eröffnet, eines diskontinuierlichen Wandels innerhalb moderner Gesellschaften. Diese können zwar anhand bestimmter struktureller Grundprinzipien von traditionalen, prämodernen (aber auch von postmodernen oder nach-modernen) Gesellschaften unterschieden werden. Doch können diese strukturellen Prinzipien auf sehr verschiedene Weise institutionalisiert sein, und dies erlaubt wiederum die Unterscheidung zwischen verschiedenen Spielarten der Moderne im historischen Vergleich, insbesondere zwischen einer Ersten und einer Zweiten Moderne. Weil die Identifizierung und genaue Definition dieser Prinzipien und Institutionen nicht nur eine theoretische Frage ist, sondern auch eine normative, weist die entsprechende Literatur eine gewisse begriffliche Ambivalenz auf. Dennoch zeigen die wesentlichen Publikationen deutlich, was die Unterscheidung im Kern ausmacht. Beck, Bonß und Lau (2001: 20 f.) zufolge beinhalten die „Prämissen der Ersten Moderne”:

- den Nationalstaat,

- eine programmatische Individualisierung innerhalb des Rahmens kollektiver Lebensmuster und Identitäten,

- Erwerbsarbeit bzw. Berufstätigkeit im Sinne der Vollbeschäftigungsgesellschaft,

- einen Begriff der Natur, der auf ihrer Ausbeutung beruht,

- ein wissenschaftlich definiertes Rationalitätskonzept,

- und das Prinzip funktionaler Differenzierung.

Ausgehend von dieser Unterscheidung zwischen Grundprinzipien und Basisinstitutionen behauptet die Theorie reflexiver Modernisierung, dass der Epochenbruch innerhalb moderner Gegenwartsgesellschaften durch die Transformation der Basisinstitutionen der Industriegesellschaft bei gleichzeitiger Radikalisierung ihrer Grundprinzipien geprägt ist. Dank des globalen Sieges der Prinzipien der Moderne (wie der Marktwirtschaft) und der unbeabsichtigten Nebenfolgen der industriellen Moderne (Klimawandel, globale Finanzkrisen) sind die grundlegenden sozialen Institutionen der Ersten Moderne für sowohl die Gesellschaft als auch die Individuen unwirksam oder dysfunktional geworden. In aller Welt zeigen die Schlüsselinstitutionen und Leitideen der Ersten Moderne: Nationalstaaten, politische Parteien, wissenschaftliche Experten, Gewerkschaften, die Demokratie, die Marktwirtschaft, die wohlfahrtsstaatlichen Sicherungssysteme, die Schulen, die Universitäten, aber auch die Familie und Geschlechterrollen immer mehr anscheinend irreversible Schwächen in der Erfüllung ihrer sozialen Funktionen und ihrer individuellen Nützlichkeit für die Stabilisierung des sozialen Handelns. Kurz, die institutionellen und normativen Selbstverständlichkeiten der industriegesellschaftlichen Moderne werden prekär. Zum Beispiel sehen sich Individuen immer öfter dazu gezwungen, ihre zerbrechlichen Erwerbs-, Liebes-, Eltern-, Geschlechter-, Familienbiographien in Kategorien individualisierter Widersprüche, Zwänge, Ziele und Lebenswege zu „meistern“, ohne auf bewährte, vorgegebene Rollenbilder zurückgreifen zu können (Beck 1983; Beck-Gernsheim 1983; Beck / Beck-Gernsheim 2001): Die individualisierte Biographie wird zum Ort, an dem die Systemwidersprüche der globalisierten Welt ausgehalten, ja im kleinen Einmaleins der in Zeitplänen, Karrieren, Elternschaften usw. abzustimmenden, individuellen Lebensführungen in gewissem Sinne sogar ,,ausgehandelt“" werden müssen. Dieser Übergang vollzieht sich keineswegs in der ganzen Welt in gleicher Weise, sondern wird in 
verschiedenen Kontexten, Pfaden, Schwellen unterschiedlich gebrochen und gespiegelt (für China siehe Yan 2010; für Japan in diesem Heft Ishida et al. 2010; für Südkorea in diesem Heft Shim / Han 2010).

Die Fallstudien über die Prozesse reflexiver Modernisierung in außereuropäischen Gesellschaften (beispielhaft in China, Japan und Südkorea) zeigen, dass neue Varianten einer Zweiten Moderne entstehen und die Pfade, auf denen sich Modernisierungsprozesse vollziehen, von Land zu Land sehr unterschiedlich sein können (siehe später). Aus diesem Grund schlagen wir vor, die Variationen der Prozesse reflexiver Modernisierung selbst zur Schlüsselvariablen in der Analyse von Transformationen moderner Gesellschaften zu machen. Genauer gesagt: Wir können mehrere Prozessvariablen identifizieren, mit deren Hilfe die Muster reflexiver Modernisierungsprozesse unterschieden werden können:

- die Handlungslogik transformativen Wandels: unbeabsichtigt versus beabsichtigt, aktiv versus reaktiv; ${ }^{4}$

- die Dauer des Transformationsprozesses: „,ausgedehnt” versus ,,verdichtet”;

- das Resultat: ,erfolgreich” versus ,,gescheitert”.

Anhand dieser Prozessvariablen können wir insbesondere unterscheiden zwischen

- dem westlichen Pfad oder Modell als Projekt einer unbeabsichtigten, zeitlich ausgedehnten und (mehr oder weniger) erfolgreichen Modernisierung moderner Gesellschaften;

- dem Projekt einer radikal beschleunigten - „compressed“ - Modernisierung (Chang 1999 und 2010 a,b), das von einem entwicklungsorientierten Staat vorangetrieben wird (Südkorea, China);

- dem Postkolonialismus als Projekt einer reaktiven, erzwungenen Modernisierung mit vielfach problematischen Folgen;

- und dem Pfad einer ,, gescheiterten” Modernisierung in dem Sinne, dass die Schaffung von Institutionen der Ersten Moderne (wie des Nationalstaates) oder die Transformation in die Zweite Moderne misslingt.

In ihrer ursprünglichen Form (vgl. etwa Beck 1986; Beck / Giddens / Lash 1996) war die Theorie reflexiver Modernisierung im Wesentlichen eine Theorie der ,westlichen” Moderne selbst. Sie hatte eine ganze Reihe von Grundannahmen mit traditionellen Theorien der Moderne und der Modernisierung gemeinsam. Unter anderem teilte sie mit ihnen den universalistischen Anspruch, indem sie davon ausging, dass ihre Normen, Prinzipien und Institutionen früher oder später weltweit Anwendung finden könnten und sollten. Diese Vorstellung muss jedoch revidiert und durch die Idee der „kosmopolitischen Modernen” ersetzt werden. Wir behaupten, dass der Prozess reflexiver Modernisierung in der Tat universell ist, dass also die institutionenverändernden Nebenfolgen industrieller Modernisierung in vielfältigen Formen beobachtbar sind. Wir behaupten dagegen nicht, dass der Prozess reflexiver Modernisierung sich überall auf dieselbe Weise und mit dem gleichen Ergebnis vollzieht. Ganz im Gegenteil: Er ist historisch-empirisch extrem kontingent. Er bringt neue Variationen moderner Gesellschaften hervor, die nicht einfach deshalb aufkommen, weil die Spielarten der Moderne sich wandeln, die Eisenstadt identifiziert hat. Der Prozess reflexiver Modernisierung bringt nicht nur neue kosmopolitische Herausforderungen hervor, sondern auch ein ganzes Spektrum von neuen Mischformen prämoderner, erst- und zweitmoderner Konstellationen. Um diese Konstellationen soziologisch adäquat analysieren zu können, bedarf es einer Erweiterung des soziologischen Horizonts. Kurz: Solange wir fortfahren, die Zweite Moderne isoliert im westlichen Kontext zu betrachten, verkennen wir sie.

4 Weil die Theorie reflexiver Modernisierung annimmt, dass sich zumindest im Fall der westlichen „Vorreiter“ der Prozess der reflexiven Modernisierung unbeabsichtigt vollzieht, können wir nicht mit Therborns Unterscheidung zwischen freiwilliger und erzwungener Modernisierung (Therborn 1995) arbeiten und benötigen ein differenzierteres Konzept von Handlungslogiken. 


\section{Kosmopolitisierung: Der Prozess, durch den sich nationale Gesellschaften gegenseitig verinnerlichen}

Unser dritter Theoriebaustein ist das Konzept der Kosmopolitisierung (Beck 2004). Wir leben nicht in einem Zeitalter des Kosmopolitismus, aber in einem Zeitalter der Kosmopolitisierung. Der ,globale Andere“ ist in unserer Mitte. Dieses Konzept der Kosmopolitisierung führt nicht einfach die ersten beiden Denkfiguren zusammen, sondern verknüpft sie derart, dass die Schwächen beider dadurch kompensiert werden. Auf der einen Seite wird die Theorie reflexiver Modernisierung für die Existenz multipler Modernen geöffnet; auf der anderen wird die Theorie multipler Modernen selbst wiederum geöffnet für die Möglichkeit diskontinuierlichen sozialen Wandels.

Unseren Ausgangspunkt bildet das Konzept des Kosmopolitismus. Die Debatte zum ,neuen" Kosmopolitismus war in den vergangenen zehn Jahren eine der wichtigsten Debatten in den Sozial- und Geisteswissenschaften. ${ }^{5}$ Im Verlauf dieser Debatte wandelte sich das Kosmopolitismus-Konzept von einer rein normativen Theorie, die in der Philosophie und der politischen Theorie beheimatet ist, zu einer sowohl normativen als auch empirischen sozialwissenschaftlichen Theorie, die in verschiedensten Disziplinen von der Anthropologie über die Geographie, die Kulturwissenschaften, die Literaturkritik, die Rechtswissenschaft, die Internationale Politik bis hin zur Sozialgeschichte breit rezipiert wurde. In unserem Zusammenhang ist ein Aspekt des Kosmopolitismus-Konzepts von besonderer Bedeutung, seine Zweidimensionalität (vgl. Appiah 2007). In der ersten, vertikalen Dimension bezieht sich Kosmopolitismus auf die individuelle oder kollektive Verantwortung gegenüber der Menschheit. In diesem Zusammenhang behauptet die Theorie reflexiver Modernisierung, dass moderne Gesellschaften - westliche ebenso wie nicht-westliche - mit qualitativ neuartigen Problemen konfrontiert sind, die „kosmopolitische Imperative” erzeugen. Diese kosmopolitischen Imperative entstehen aus globalen Risiken: ökologischen, technologischen und ökonomischen Risiken, die von unzureichend regulierten Märkten herrühren oder aus den „Errungenschaften“ neuer Risikotechnologien (Kernenergie, Humangenetik usw.) hervorgehen. Diese neuen globalen Risiken erzeugen Ketten miteinander verzahnter politischer Entscheidungen zwischen Staaten und ihren Bürgern, die bzw. deren Folgen den Charakter und die Dynamik territorial definierter Herrschaftssysteme von innen heraus verändern. Diese Risiken verbinden den globalen „Norden“ und den „Süden“ in zuvor nicht gekannter Weise. Das Resultat dieser globalen Verflechtungen ist jedoch nicht der normative Kosmopolitismus einer „Welt ohne Grenzen“. Vielmehr bringen besagte Risiken eine neue kosmopolitische Verantwortung gegenüber der Menschheit hervor, einen kosmopolitischen Imperativ, dem sich niemand entziehen kann. Daraus entsteht die universelle Möglichkeit von (nationale) Grenzen übergreifenden „Risikogemeinschaften”, die unvermittelt auftauchen, durch strategisches Handeln etabliert und sich ihrer kosmopolitischen Zusammensetzung bewusst werden können - ,imagined cosmopolitan communities”.

5 Zu den wichtigsten Beiträgen zu dieser Debatte zählen: Appiah 2007; Archibugi 2008; Baban 2006; Beck 2002, 2004, 2007; Beck / Grande 2004, 2010; Beck / Sznaider 2006 a,b,c, 2010; Beitz 2005; Benhabib 2007; Berry 2008; Boon / Fine 2007; Braeckman 2008; Brassett / Smith 2007; Bray 2009; Breckenridge et al. 2002; Brock / Brighouse 2005; Brown 2008; Calcutt / Woodward / Skrbis 2009; Calhoun 2007 a, 2007 b; Cheah 2006; Delanty 2009; Delanty / He 2008; Dobson 2006; Edwards 2008; Eriksen 2009; Featherstone et al. 2002; Fine 2007; Garsten 2003; Grande 2006; Hannerz 2004; Held 2010; Inglis 2009; Kendall / Woodward / Skrbis 2009; Khagram / Levitt 2008; Kurasawa 2004; Levy / Heinlein / Breuer 2010; Levy / Sznaider 2007, 2010; Mau / Mewes / Zimmermann 2008; MeckledGarcia 2008; Mendieta 2009; Nederveen Pieterse 2006; Nowicka / Rovisco 2009; Pichler 2008; Poferl / Sznaider 2004; Rapport 2007; Rumford 2007; Slaughter 2009; Todd 2007; Tyfield / Urry 2010; Vertovec / Cohen 2002; Werbner 2008; Wimmer / Glick Schiller 2002; Ypi 2008; Zhang 2010; siehe auch die Themenhefte folgender Zeitschriften: British Journal of Sociology 2006, Heft 57 / 1 und 2010, Heft 61 / 3; Constellations 2003, Heft 10 / 4; Daedalus 2008, Heft 137 / 3 und The Hedgehog Review 2009, Heft $11 / 3$. 
Sie entstehen aus der Einsicht, dass zivilisatorische Gefahren und Risiken nicht mehr sozial in Raum oder Zeit eingegrenzt werden können. Im Angesicht dieser kosmopolitischen Imperative muss eine neu formulierte Theorie reflexiver Modernisierung das Argument entfalten, dass wir inzwischen alle in einer Zweiten, kosmopolitischen Moderne leben - unabhängig davon, ob ,wir“ niemals modern waren oder schon länger modern sind.

In seiner zweiten Dimension ist Kosmopolitismus eine Theorie der Vielfalt, oder genauer, einer bestimmten Weise, Vielfalt zu deuten und mit ihr umzugehen (Delanty 2009; Tyfield / Urry 2010 in diesem Heft). In diesem Zusammenhang von kosmopolitischer Moderne zu sprechen heißt, unseren Horizont so zu erweitern, dass wir ein ganzes Spektrum westlicher und nicht-westlicher Modernen in den Blick bekommen. Die konzeptuelle Herausforderung, vor der eine Theorie kosmopolitischer Modernisierung damit steht, ist, die Muster der Variation sowie ihre Ursachen und Folgen zu identifizieren. Kurz: Die Idee kosmopolitischer Moderne muss aus einer Vielheit von Modernen entwickelt werden, aus dem inneren Reichtum der Spielarten der Moderne. Kosmopolitische Modernisierung darf jedoch nicht mit dem Konzept der Pluralisierung gleichgesetzt werden. Sie unterstreicht nicht nur die Existenz einer Vielfalt verschiedener Typen moderner Gesellschaften, sondern betont auch die dynamische Verknüpfung und Interaktion von bzw. zwischen Gesellschaften. In dieser Hinsicht übernimmt sie wichtige Konzepte aus der Literatur zum Postkolonialismus, etwa „Verwobenheit” (, entanglement”; Randeria 1999, 2004), und zur Globalisierung, etwa „Vernetzung” (,, interconnectedness"; Held et al. 1999) und führt diese durch die Einführung des Konzepts der „Verinnerlichung des Anderen" einen entscheidenden Schritt weiter. Die Kosmopolitisierung verbindet Individuen, Gruppen und Gesellschaften und setzt sie über bestehende Grenzen und Dualismen hinweg auf eine neue Art in Beziehung zueinander, wodurch Stellung und Funktion des „Selbst” und des „Anderen” einen Wandel erfahren. Eine solche „Verinnerlichung des globalen Anderen” (,the global other is in our midst") macht die soziologische Konsequenz, das „soziologische Gesicht“ der Globalisierung aus und sichtbar. Zentral ist ferner: Kosmopolitisierung kann aus zwei völlig verschiedenen Prozessen hervorgehen. Zum einen kann sie das Resultat einer aktiven, bewussten und reflexiven Öffnung von Individuen, Gruppen und Gesellschaften für fremde Ideen, Präferenzen, Regeln und kulturelle Praktiken sein; zum anderen kann sie aber auch Ergebnis passiver und ungeplanter Prozesse sein, die eine Verinnerlichung der Andersheit erzwingen. Daher ist Kosmopolitisierung definitionsgemäß kein symmetrischer und autonomer Prozess; sie kann durchaus das Produkt von Asymmetrien, Abhängigkeiten, Macht und Gewalt sein, und sie kann auch neue Asymmetrien und Abhängigkeiten in und zwischen Gesellschaften erzeugen.

Integriert man diese zwei Dimensionen der Kosmopolitisierung, so wird deutlich, dass kosmopolitische Modernen sich erheblich von einer Kantischen Welt des ,ewigen Friedens” unterscheiden. Sie sind vielmehr von einer widersprüchlichen Dynamik geprägt, die man als kosmopolitische Dialektik beschreiben und begreifen kann. Einerseits vollzieht sich ein zentripetaler, einigender Prozess; die Bildung eines gemeinsamen Verantwortungshorizontes der Weltrisikogesellschaft und zwar nicht als normative Setzung, sondern als epochales Faktum (Beck 1999, 2007). Zugleich aber ist die Zweite Moderne von mächtigen zentrifugalen, diversifizierenden Prozessen gekennzeichnet, die sich aus der Koexistenz, vielleicht sogar dem Hegemonialkampf zwischen verschiedenen Typen und Visionen der Moderne sowie aus Widerständen gegen ökonomische, politische und kulturelle Globalisierung innerhalb von Gesellschaften ergeben (vgl. Kriesi et al. 2008).

Es ist also - noch einmal hervorgehoben - klar zwischen normativ-philosophischem - ,reinem“- Kosmopolitismus und real existierender - ,unreiner" - Kosmopolitisierung zu unterscheiden, wobei letztere zum Thema der empirisch-theoretischen Sozialwissenschaft wird. Kosmopolitisierung meint eine reflexive conditio humana, in der sich die scheinbar ontologisch 
bestimmte Vorstellung »getrennter Welten«, die Unterscheidung zwischen »Wir« und »die Anderen«, nicht länger anwenden läßt. Die Opposition zwischen einem »Wir«, das hier lebt, und »die Anderen«, die dort leben, die geographische, kulturelle, gesellschaftliche und politische Trennung zwischen dem »Eigenen« und dem »Fremden« zerbricht de facto. Im Risikound Internetzeitalter wird die Welt zum alltäglichen Bezugshorizont, zum Raum neuer Ungleichheiten und neuer Handlungsmöglichkeiten - unabhängig davon, ob die Akteure dies wissen, wollen, nutzen oder erleiden und als Verlust von Autonomie und Identität beklagen und bekämpfen. Alle Akteure - Migranten, Konzerne, religiöse Gemeinschaften, Menschenrechtsbewegungen, Wissenschaftler, Arbeiter, Lehrer, Ärzte, Klimaforscher, Mitglieder von »Welt-Familien «, aber auch Kriminelle und nicht zu vergessen Neonationalisten und Al-Qaida-Terroristen - müssen ihren Wahrnehmungs- und Aktionshorizont erweitern, aktiv vergleichen, fremde Perspektiven einnehmen und für die eigenen Zwecke koordinieren: Das meinen wir mit reflexiver conditio humana. Dieser historisch neuartige Zwang zur Reflexion, zum Blickwechsel über Grenzen hinweg, oft auch mit Bezug auf das globale Gemeinwohl, darf jedoch nicht mit Normativität, mit den damit auch aufgeworfenen, großen Fragen der philosophischen Ethik - was ist »human«? was ist »Humanität«? -, verwechselt werden. Das wäre ein versteckter Hegelianismus, ein eklatanter Fehlschluss und zwar von dem epochalen Faktum der „unreinen“ Kosmopolitisierung auf die Normativität des „reinen“ Kosmopolitismus.

Um im Beispiel zu sprechen: Die »humanitäre Interventionen«, die dem Menschenrechtsregime mit militärischen Mitteln zur Durchsetzung verhelfen sollen, bilden einen realen Bestandteil von Weltinnenpolitik, obwohl die normativ-philosophischen wie politisch-rechtlichen Institutionen erst in Bruchstücken vorliegen. Es gibt keine (wirklich wirksame) Weltgerichtsbarkeit, keine global operierenden Kräfte, die wie eine Weltpolizei in anderen Staaten intervenieren könnten, und keine Mittel, um demokratische Staatenbildung zu gewährleisten.

Der entscheidende Punkt ist: Die Diagnose der real existierenden Kosmopolitisierung hat also keine Antwort auf die ethische Mammutfrage: Sind - oder genauer: unter welchen Bedingungen sind - humanitäre Interventionen gerechtfertigt, verwerflich oder geboten? Aber sie beantwortet die Frage, warum die Frage nach der Rechtfertigung humanitärer Interventionen weltweit auf der Agenda steht und warum sie weniger einen Lernprozess in Gang setzt als vielmehr mannigfaltige Konflikte hervorruft. ${ }^{6}$

\section{De-Konstruktion und Neudefinition westlicher Moderne an den Beispielen von Individualisierung und Risikogesellschaft}

Die „kosmopolitische Wende“ in der soziologischen Theorie wirft die Frage auf, was wir aus den Diskursen über Variationen der Zweiten Moderne und insbesondere aus den außereuropäischen Erfahrungen und Perspektiven für die soziologische Theorie im Allgemeinen lernen können. Im Folgenden werden wir zeigen, dass die Grundbegriffe der Theorie der Zweiten Moderne - wie jeder anderen Gesellschaftstheorie - nicht so ohne weiteres „reisefähig“ sind, also in verschiedenen Kontexten und Kontinenten einfach ,angewandt" werden können und ebenso wenig im Weltmaßstab funktionieren. Sie müssen de-konstruiert und re-definiert werden, um in unterschiedlichen sozialen und historischen Situationen brauchbar zu sein. Deshalb schließt die kosmopolitische Wende eine Selbstkritik der universalistischen Annahmen und Erwartungen der frühen Theorie reflexiver Modernisierung ein: Die Theorie reflexiver Modernisierung muss sozusagen selbst kosmopolitisiert werden. Hier stellen sich folgende Fragen: Wie können grundlegende Konzepte von „Individualisierung“ und „Risiko“ an eine Moderne angepasst werden, die sich auf verschiedenen Pfaden vollzieht? Welche Folgen haben

6 Zur aktuellen Diskussion um „Kosmopolitisierung“ siehe u.a. Beck / Beck-Gernsheim 2011; Chang 2010 a,b; Calhoun 2010; Gilroy 2010; Levy et al. 2010; Maharaj 2010; Sznaider 2010; Turner 2010; Tyfield / Urry 2010; Zhang 2010. 
die ostasiatischen, südamerikanischen usw. pfadabhängigen Variationen (der Zweiten Moderne) für die Theorie der Zweiten Moderne im Allgemeinen?

\section{Neudefinition des europäischen Individualisierungsmodells}

Mit den ,verwobenen“ Fällen und Kontexten des japanischen (Ishida et al. 2010) und südkoreanischen (Shim / Han 2010) Pfades zur Individualisierung präsentiert dieses Heft konzeptionell und empirisch reichhaltige Ressourcen für die Dekonstruktion und Neudefinition des europäischen Individualisierungsmodells. Die Individualisierungsthese (vgl. Bauman 2001; Beck 1983; Beck / Beck-Gernsheim 1994, 2001; Beck-Gernsheim 1983; Giddens 1991) hebt vier Hauptmerkmale des Individualisierungsprozesses hervor: (1) die Enttraditionalisierung; (2) die institutionalisierte Herauslösung und Wiedereinbettung des Individuums; (3) den Zwang zum Verfolgen eines ,eigenen Lebens“ und das Fehlen einer genuinen Individualität, sowie (4) die Verinnerlichung von systemischen Risiken. Dieses Individualisierungstheorem beruht auf zwei Grundannahmen: Theoretisch versteht es sich als Gegenthese zum Neoliberalismus und implizit auch zum Liberalismus und klassischen Individualismus; sozialwissenschaftlich definiert es den Individualisierungsprozess unter den Bedingungen kultureller Demokratie und des Wohlfahrtsstaates. Diese Annahmen verweisen jedoch weitgehend auf die Geschichte und Wirklichkeit (West)Europas; sie legen das Individualisierungstheorem auf die Sonderform der Zweiten Moderne Europas fest (bzw. beschränken seine Geltung auf Westeuropa). Wenn man die zwei Annahmen jedoch von den vier Hauptmerkmalen entkoppelt, dann wird erkennbar, dass die Individualisierung ein globaler Trend der Gegenwart ist. Einerseits prägen sich die vier Merkmale in unterschiedlichen Modernisierungspfaden auf verschiedene Weise aus. Andererseits zeigt der globale Individualisierungstrend auch Merkmale, die nicht auf die genannten zwei Grundannahmen reduziert werden können (Yan 2010).

Im europäischen Modell ist die Hoffnung eingewoben, dass die instrumentellen Beziehungen kapitalistischer Märkte selbst unter post-wohlfahrtsstaatlichen Bedingungen eine Art Individualisierung hervorgebracht haben, die mit Ideen kultureller Demokratisierung verwoben ist. Dagegen gilt dies beispielsweise für die chinesische Individualisierung eindeutig nicht. In China findet man weder eine kulturell eingebettete Demokratie noch einen Wohlfahrtsstaat. Überdies besitzt die Individualisierung in China im Gegensatz zur europäischen keine institutionelle Verankerung in einem System von Grundrechten (Familienrecht, Arbeitsrecht usw.).

Wie man sieht, ist das, was im europäischen Kontext als „universelle Logik“ der Individualisierung angesehen wird - nämlich das Aufeinandertreffen der institutionellen, rechtlichen Formen und der biographischen Muster der Individualisierung -, in Wirklichkeit eine historisch und kulturell begrenzte Spezialform, das Resultat einer partikularen Amalgamierung von Modernisierung und Individualisierung im europäischen Zusammenhang. Wie das chinesische Beispiel zeigt, können diese zwei Entwicklungen - Rechtsformen und biographische Muster - auch entkoppelt und rekombiniert werden, wobei grundverschiedene Pfade der Individualisierung entstehen.

Prinzipiell besteht sowohl im europäischen als auch im chinesischen Kontext eine enge Verbindung zwischen Individualisierung und Staat. Diese Verbindung kann jedoch völlig verschiedene Formen annehmen, sie kann in der Tat in diametral entgegengesetzte Richtungen weisen. Wenn auch in China das Individuum an Bedeutung gewinnt, dann geschieht das nicht in einem institutionell gesicherten Rahmen oder auf Grundlage bürgerlicher, politischer und sozialer Grundrechte, die im Europa der Ersten Moderne politisch erstritten wurden. Diese Rechte sind dort noch immer umkämpft, und das mit ungewissem Ausgang.

Es fällt auf, dass der chinesische Pfad der Individualisierung im Vergleich zum europäischen eine charakteristisch andere, in der Tat umgekehrte Entwicklungssequenz aufweist. In China 
wird die neoliberale Deregulierung der Wirtschaft und des Arbeitsmarktes, der Alltagskultur und des Konsums vor der und ohne die institutionelle Verankerung der Individualisierung betrieben, wie wir sie in Europa kennen. Daher müssen politische und soziale Grundrechte auf der Basis neoliberaler, depolitisierender, marktförmiger Individualisierung gewonnen werden. Eine Folge dieser Umkehrung besteht darin, dass der autoritäre Staat, der seine sozialen Garantien und Verpflichtungen gegenüber dem Kollektiv widerrufen hat, dem Verlangen nach politischer Teilhabe, das dem Prozess der Individualisierung innewohnt, Grenzen zu setzen versucht, indem er ein enges Netz von Kontrollen um das Individuum spannt. Individualrechte werden als Privilegien gewährt, nicht als unverletzliche Grundrechte, die jeder Bürger besitzt. Durch ihre Verknüpfung mit den offiziell hochgehaltenen nationalen und familiären Werten versucht die Regierung, die von ihr selbst benötigte Individualisierung zu beschränken. Zusammenfassend lässt sich sagen, dass in Europa Recht und Gesetz die Sprache der Individualisierung sprechen, während sich in China eine Praxis der tolerierten, sogar forcierten Individualisierung etabliert, verbunden jedoch mit ihrer offiziellen ideologischen Stigmatisierung. Es ist unschwer zu erkennen, dass derartige ostasiatische Erfahrungen und Perspektiven der Zweiten Moderne die Begriffsarbeit enorm bereichern (siehe auch Ishida et al. 2010 sowie Shim / Han 2010 in diesem Heft).

\section{Neudefinition des europäischen Modells der Risikogesellschaft}

Die Notwendigkeit einer Neudefinition von Grundbegriffen der Gesellschaftstheorie zeigt sich auch am Konzept des Risikos. ${ }^{7}$ Aus ihrer Untersuchung verschiedener Typen von Risiken im Zusammenhang mit der ostasiatischen „compressed modernization” (Chang 1999) schlussfolgern Han und Shim, dass ,das Konzept der Risikogesellschaft auf Ostasien besser zutrifft als auf westliche Gesellschaften" (Han / Shim 2010) - aber neu definiert werden muss. Sie unterscheiden zwei Arten der Risikoproduktion in Gegenwartsgesellschaften. Auf der einen Seite werden „neue, globale Risiken” erzeugt, die aus der Radikalisierung der Ersten Moderne hervorgegangen sind. Diese sogenannten ,manufactured uncertainties” (Beck 1996; Giddens 1997) sind der vorherrschende Typ des Risikos in westlichen, insbesondere westeuropäischen Gesellschaften. Zu ihnen gehören der Klimawandel, der transnationale Terrorismus und die systemischen ökonomischen Risiken. „Es sei jedoch falsch“, so Han und Shim, ,alle Risiken in dieser Weise zu konzeptualisieren" (Han / Shim 2010). Gibt es doch zugleich bestimmte Arten von Risiken, die als Folgen jener Defizienzen entstehen, die in besonderer Weise der ostasiatischen ,compressed modernization“ zuzurechnen sind. Beispiele für diese „DefizienzRisiken" sind Großunfälle verschiedener Art, Gewaltausbrüche, Kontaminationen von Nahrung und Trinkwasser, Bestechung, Betrug, Verfall der Familie usw.

Han und Shim unterscheiden des Weiteren zwei verschiedene Arten der Verbreitung von Risiken: eine transnationale und eine regionale: „Risiken können ,transnational ' genannt werden, wenn sie im Prinzip überall auf der Welt auftreten können. Im Gegensatz dazu können sie ,regional' sein, wenn sie vornehmlich in bestimmten Ländern in Erscheinung treten, die einen bestimmten, gemeinsamen Pfad in die Moderne eingeschlagen haben” (ebd.). Die „Defizienzrisiken”, die durch die rasante ,compressed“ Modernisierung in Ostasien entstehen, sind im Wesentlichen von regionaler Reichweite. Sie wirken sich auf ostasiatische Länder in spezifischer Weise aus, obwohl sie sich in globalem Kontext materialisieren. In der Konsequenz nimmt die Risikogesellschaft in ostasiatischen Ländern eine völlig andere Form an als in Westeuropa. Sie konstituiert sich aus anderen Risiken, anderen Risikokulturen und anderen Risikokonflikten. Han und Shim schließen daraus, dass sich die Beschäftigung mit der „Weltrisikogesellschaft” zentral auch auf die verschiedenen „Provinzen“, die konkrete Wirklichkeit

7 Einen hervorragenden Überblick über die verschiedenen soziologischen Konzepte des Risikos bieten Arnoldi (2009 a;b) und Wilkinson (2010). 
Ostasiens einlassen muss, und zwar nicht deshalb, weil der westliche Fokus auf globalen Risiken nicht zulässig wäre, sondern weil andere Arten von Risiken, die gerade aus der Logik der ,,compressed modernization“ herrühren, in Ostasien ebenso dringend nach Aufmerksamkeit verlangen (ebd.).

Um diese Unterscheidung zwischen Arten der Risikoproduktion anhand von Modernisierungserfolgen und -defizienzen weiterzuentwickeln, schlagen wir vor, zwischen, selbst-induzierten" und ,, fremd-induzierten" Gefahren zu unterscheiden. Damit wird die Ungleichheitsund Herrschaftsproblematik im Konzept globaler Risiken selbst verortet. Auf diese Art und Weise wird es möglich, präziser als bisher zu erfassen, dass die Lagen der Verursacher und der Betroffenen von Risken bzw. Gefahren radikal ungleich aufeinander bezogen sind. Spätestens mit der kosmopolitischen Wende wird dann erkennbar, dass die Unterscheidung zwischen Selbstgefährdung und Fremdgefährdung insofern von kosmopolitischer Brisanz ist, als das Verhältnis ganzer Weltregionen zueinander als Herrschaftsverhältnis der Externalisierbarkeit von selbst-produzierten Gefahren auf Andere analysierbar wird: Die Mächtigen produzieren Risiken und profitieren von ihnen, während die Anderen, die Ohnmächtigen, bis in das Mark ihrer Existenz hinein von den Nebenfolgen der Entscheidung Anderer betroffen sind, ohne jegliche Chance, an deren Entscheidungen teilzuhaben.

Hier von „Klassen“ zu sprechen verdeckt und verfehlt allerdings die wesentliche Differenz: Diese radikalen Ungleichheiten in der Weltrisikogesellschaft entstehen nicht aus der Konkurrenz um knappe Ressourcen - um „goods“, sondern eben aus der Externalisierbarkeit von „, bads “ sowie aus der globalen Interdependenz von regional, oft im westlichen Kontext entstandenen Globalrisiken (Beck / Poferl 2010). Allein diese noch sehr grobe Unterscheidung von Selbstgefährdung und Fremdgefährdung erlaubt, erzwingt eine Revision der Theorie der Risikogesellschaft. Die Prämisse, dass alle Menschen und Gesellschaften der Welt die Risiken, die sie existentiell bedrohen, als Nebenfolgen ,eigener"Entscheidungen (und damit im engeren Sinne als „Risiken“) verstehen, muss preisgegeben werden zugunsten der Annahme, dass in großen Teilen der Erdbevölkerung die Modernisierungsfolgen als fremd-induzierte Gefahren anderen Entscheidern in anderen Erdteilen - sprich: dem Westen - zugerechnet werden (oder auch umgekehrt, wie aus dem Zurechnungskonflikt um die Folgen des Klimawandels hervorgeht). Sozial radikal ungleich betroffen sind derartige Regionen damit allerdings nicht länger nur durch die Folgen gescheiterter Modernisierung (wie beispielsweise Dependenztheoretiker unterstellen) sondern auch durch katastrophale Nebenfolgen erfolgreicher Modernisierungsprozesse.

An dieser Stelle bietet der Begriff der Weltrisikogesellschaft einen forschungsstrategischen Vorteil. Gibt er uns doch einen klaren Anhaltspunkt für eine entscheidende „Nahtstelle“ zwischen Konflikt und Integration: die Janusköpfigkeit globaler Risiken. Zum einen entstehen in den diskursiven Räumen globaler Risiken „,imagined cosmopolitical communities“, die die nationalen imagined communities, die Benedict Anderson (1996) so brillant analysiert hat, zwar nicht ersetzen oder zersetzen, aber doch unter dem Druck der kosmopolitischen Schicksalsgemeinschaft für einander öffnen und kooperativ vernetzen können (nicht müssen). Zum anderen entzünden sich gerade an den transnationalen Risikoungleichheiten - an den Maßstäben von Betroffenheit und sozialer Verwundbarkeit - wiederum grenzübergreifende RisikoZurechnungskonflikte, die sich einer globalen Vereinheitlichung nachhaltig widersetzen. Zugleich versagen die für die Zivilisierung von Klassenkonflikten vorgesehenen Ressourcen, handelt es sich doch um antizipierte zukünftige Katastrophen, die - das ist ein entscheidender Punkt - gerade durch legales Handeln in Gang gesetzt werden. Wenn man von der thematisch und zeitlich schwer eingrenzbaren, vorweg eilenden Politik der Prävention absieht, gibt es wenig Ressourcen und Spielräume, um antizipierte, ,legale“ Zukunftskatastrophen ,umzuverteilen“. 


\section{Theoretische Implikationen}

Was sind die allgemeineren theoretischen Implikationen dieser exemplarischen De-Konstruktion und Re-Konstruktion soziologischer Grundbegriffe? Die erste Einsicht ist, dass die Abfolge von der Prämoderne über die Erste bis hin zur Zweiten Moderne nicht universell ist und nicht verallgemeinert werden kann. Im Gegenteil, diese Sequenz ist eine zentrale Eigenschaft des speziell europäischen Pfads zur Moderne. Man kann den falschen Universalismus soziologischer Theorien nicht aufdecken, indem man aus europäischer Sicht auf Europa schaut. Man „sieht” ihn nur aus außereuropäischer Perspektive, also mit ,,asiatischen Augen” (oder afrikanischen usw.), oder mit anderen Worten, indem man methodologischen Kosmopolitismus praktiziert. Dieser nämlich beinhaltet nicht nur die Erfahrungen und Sichtweisen anderer bezüglich der Modernisierung, sondern unterzieht das Selbstverständnis der europäischen Moderne einer Korrektur und Neudefinition. Die Frage, wie Variationen der Zweiten Moderne beschaffen sein können, findet hier eine systematische Antwort: in Begriffen verschiedener Abfolgen, Kombinationen und Mischungen von Prämoderne, Erster und Zweiter sowie NachModerne. Vor allem können „spät"kapitalistische Gesellschaften anhand der immanenten, selbstverursachten Konsequenzen des Prozesses reflexiver Modernisierung präziser charakterisiert werden, nämlich durch die Transformation von der Ersten in die Zweite Moderne, in der sich grundlegende Institutionen der Ersten Moderne unterminieren. In diesem Fall entstehen die meisten Triebkräfte der radikalisierten Reflexivität - Individualisierung, Kosmopolitisierung und Risikogesellschaft - als „Nebenfolgen” selbst oder fremd geschaffener Risiken. In gleicher Weise lassen sich Entwicklungsländer in späteren Stadien, in rapider Entwicklung begriffene kapitalistische Gesellschaften und sozialistische Schwellenländer anhand verschiedener Arten von Zeit-Raum-Differenzierungen, Kombinationen und Verbindungen der PräModerne, der Ersten und der Zweiten Moderne charakterisieren und unterscheiden.

Theoretisch können in diesem Zusammenhang zusätzlich zum europäischen Muster gesellschaftlicher Modernisierung zwei Konstellationen unterschieden werden: zum einen die der europäischen entgegengesetzte Sequenz, in der die Herausforderungen der Zweiten Moderne vor der Ersten Moderne auftreten und daher deren institutionelle Ressourcen fehlen. Dies könnte als die „Opfer-Konstellation fortgeschrittener Entwicklungsländer” bezeichnet werden. Der zweite Typ wäre die Konstellation der ,compressed“ Modernisierung (Chang 1999), in der die Entwicklung der Ersten Moderne (die im europäischen Kontext auf mehr als 150 Jahre ausgedehnt war) und der Übergang zur Zweiten Moderne (also die Prozesse der Individualisierung, des Risikos und der Kosmopolitisierung) sich plötzlich und nahezu gleichzeitig innerhalb der sehr kurzen Zeitspanne von einem oder zwei Jahrzehnten vollziehen (wie im Fall Südkoreas oder auch Chinas). Natürlich folgen die ,,ausgedehnte” europäische und die „,compressed" ostasiatische Modernisierung sehr verschiedenen Logiken. Erstens multiplizieren sich die kumulativen Effekte; zweitens sind die politischen Risiken der Modernisierung, die aus dem Kreislauf von öffentlicher Leugnung und Hysterie entstehen, in diesen zwei Varianten der Zweiten Moderne sehr unterschiedlich; drittens und daraus folgend bedeutet „Reflexivität” in beiden Zusammenhängen etwas recht Verschiedenes.

Alle diese Modernisierungspfade sind mit dem Problem konfrontiert, welche Art und Qualität von Sicherheit Individuen, Gruppen und Klassen geboten werden kann, um mit den Risiken, Unsicherheiten, Ungewissheiten und Bedrohungen zurechtzukommen, die mit sozialen Transformationen einhergehen. Im Wesentlichen können drei Typen von Risikoversicherung theoretisch unterschieden werden: ein etatistisches Modell, in dem der Staat Risikovorsorge bietet, ein gesellschaftliches Modell, in dem verschiedene sozialen Institutionen (Familien, Firmen usw.) Risiken auf sich nehmen, und ein neoliberales Modell, in dem das Individuum mit Risiken selbst zurechtkommen muss. Das etatistische Modell findet sich beispielhaft im europäischen Wohlfahrtsstaat, der aus den Klassenkonflikten der Industrialisierung hervorge- 
gangen ist (Ewald 1993). ${ }^{8}$ Japan repräsentiert eher das gesellschaftliche Modell der Risikovorsorge (Ishida et al. 2010 in diesem Heft).

Allgemein gesprochen: Es gibt eine globale Dynamik reflexiver Modernisierung, die pfadabhängige Funktionalitäten von Institutionen der Ersten Moderne auflöst. Seit Beginn des 21. Jahrhunderts mussten die Menschen vieler Länder feststellen, dass grundlegende Institutionen ihres wirtschaftlichen, sozialen und politischen Lebens plötzlich ineffektiv oder unzuverlässig wurden oder gar in einen Prozess der Selbstauflösung oder sogar Selbstzerstörung eintraten. Aufgrund der verschiedenen Mischungen von Defizienzen ebenso wie Erfolgen und Misserfolgen der nationalen, industriellen, kapitalistischen Modernisierung in verschiedenen Weltregionen sind daraus verschiedene Modernisierungspfade, Muster der Risikogesellschaft und Typen von Zweiter Moderne entstanden. Eine sowohl theoretisch als auch empirisch völlig offene Frage ist, ob und wie diese verschiedenen Typen von Zweiter Moderne problemlos koexistieren können.

\section{Methodologischer Kosmopolitismus: Das Problem der Untersuchungseinheit}

Eine Schlüsselfrage, die die kosmopolitische Wende in den Sozialwissenschaften aufwirft, lautet: Welche Untersuchungseinheit wird der Gesellschaftstheorie und -forschung zugrunde gelegt? Oder genauer: Wie können Untersuchungseinheiten jenseits des methodologischen Nationalismus gefunden und festgelegt werden, die es erlauben, die komplexen Prozesse und Interdependenzen der Kosmopolitisierung zu erfassen und Variationen kosmopolitischer Modernen vergleichend zu analysieren? Worauf kann man die sozialwissenschaftliche Analyse beziehen, wenn man sie einerseits aus dem „Container“ des Nationalstaats befreien, andererseits aber nicht zu abstrakten Konzepten der „Weltgesellschaft“ Zuflucht nehmen will? Im vorangegangenen Kapitel haben wir bereits erste Beispiele für einen methodologischen Kosmopolitismus vorgestellt. An die Stelle von Nationalstaaten bzw. nationalen Gesellschaften wurden

(a) gesellschaftstheoretisch begründete, inhaltlich-thematische Einheiten gesetzt - in unserem Fall „Individualisierung“ und „Risikogesellschaft" -, die es erlauben, in historischen und vergleichenden Analysen die Pfadabhängigkeit dieser Theoreme und ihre gesellschaftliche Spezifik zu dekonstruieren und zu redefinieren.

(b) Dabei lassen sich neue handlungs-, konflikt- und integrationsrelevante Formen von Dependenz, Interdependenz und ,interconnectedness" unterscheiden und entfalten, wie wir soeben anhand der Frage nach dem Verhältnis von Selbstgefährdung und Fremdgefährdung durch globale Risiken exemplarisch vor Augen geführt haben.

Diese Beispiele zeigen bereits wichtige Anhaltspunkte dafür auf, wie die Probleme des methodologischen Nationalismus gelöst werden können. Die empirische Forschung in so unterschiedlichen Fächern wie der Soziologie, der Ethnologie, der Anthropologie, der Geographie, oder der Politikwissenschaft hat in den vergangenen Jahren eine große Zahl von Konzepten entwickelt, die alle das Ziel haben, die vermeintlich ,natürliche“ Gleichsetzung von „Gesellschaft / Nation / Staat“ aufzubrechen. Paul Gilroys Konzept des „Black Atlantic“(1993), Saskia Sassens Identifzierung der „Global City“ (1991, 1996), Arjun Appadurais Auffassung von „scapes“ (1996, 1998), Martin Albrows Konzept des „Global Age“ (2007) und unsere eigene

8 Die ,drei Welten des Wohlfahrtskapitalismus”, die Esping-Andersen (1990) herausgearbeitet hat, variieren abhängig von Zuschnitt und Ausmaß öffentlicher Risikovorsorge und der speziellen Arten der Lastenteilung zwischen Staat und Individuum. Dies sind jedoch nur Spielarten des etatistischen Modells. 
Analyse des „, kosmopolitischen Europas“ (Beck / Grande 2004) sind Beispiele für diese Forschungsrichtung.

Diese Vielfalt von Konzepten und Ansätzen lässt sich mit Hilfe von zwei Unterscheidungen sinnvoll systematisieren. Zum einen lassen sich neue Untersuchungseinheiten danach unterscheiden, ob sie sich auf Prozesse der Transnationalisierung beziehen oder auf transnationale Strukturen. Ein Beispiel für Ersteres wären transnationale Migrationsprozesse, ein Beispiel für Letzteres wären ,diaspora communities“, in denen sich Migranten neue Formen des transnationalen Zusammenlebens schaffen. Eine zweite Unterscheidung bezieht sich auf die Reichweite und die Verortung der Kosmopolitisierung. Bei der Reichweite geht es um die Frage, welche Rolle das Nationale, d.h. der Nationalstaat, nationale Kulturen, etc., im Zeitalter der Kosmopolitisierung (noch) spielen. Bei der Verortung geht es darum, auf welcher Ebene der Fokus der Kosmopolitisierung gelegt wird - oberhalb des Nationalen: Weltregionen, Weltreligionen; unterhalb des Nationalen: das Lokale, die Familie, das Individuum, das Unternehmen, die Arbeit etc. Für den methodologischen Kosmopolitismus von besonderer Bedeutung ist die Frage nach dem Stellenwert des Nationalen und des Nationalstaats bei der Bestimmung von Untersuchungseinheiten. Die methodologisch radikalste Möglichkeit besteht darin, dass die nationale Rahmung der Untersuchungseinheit ersetzt wird durch andere Foki und das Nationale dadurch gänzlich obsolet oder irrelevant wird (,replacing the national“). Wenn man den methodologischen Nationalismus darauf beschränken würde, würde man seine Reichweite und seine Anwendungsmöglichkeiten unzulässig einschränken. Denn die empirische Globalisierungsforschung hat längst gezeigt, dass der Nationalstaat auch im Zeitalter der Globalisierung nicht gänzlich verschwinden wird. Deshalb ist es sinnvoll, eine weitere Möglichkeit in Betracht zu ziehen. Diese besteht darin, dass der Nationalstaat und das Nationale zwar weiterbestehen, dass sie aber ihre epistemologische Monopolstellung verlieren, zum Beispiel weil sie integriert werden in neue Formen der politischen Organisation und der gesellschaftlichen Ordnung. Die methodologische Konsequenz bestünde dann darin, neue Untersuchungseinheiten zu finden, in denen das Nationale zwar enthalten ist, die aber nicht mehr deckungsgleich sind mit dem Nationalen. Wir würden dies als „Einbettung des Nationalen“ (embedding the national) bezeichnen.

In diesem Zusammenhang muss betont werden, dass sich aus einer unveränderten oder gar neu wachsenden Bedeutung des Nationalstaates im Zeitalter der Globalisierung keine Rechtfertigung für einen methodologischen Nationalismus formulieren lässt. Dem liegt ein Fehlschluss von der Akteurs- auf die Beobachterperspektive zugrunde. Selbst wenn der Nationalstaat funktional an Bedeutung gewinnen würde, so muss er doch seine epistemologische Monopolstellung als die maßgebliche Untersuchungseinheit verlieren, weil die empirische Erforschung des Kosmopolitisierungsprozesses nur dann gelingen kann, wenn andere Foki ins Zentrum gerückt werden und der Nationalstaat von der konstant vorgegebenen Rahmung zur empirischen Variable wird (vgl. Zürn 2001).

Die folgende Tabelle gibt einen Überblick über die vier Varianten des methodologischen Kosmopolitismus, die sich aus diesen beiden Unterscheidungen ergeben. Für jede der Varianten haben wir ausgewählte Beispiele aus der Literatur zu Kosmopolitismus, Transnationalismus und Globalisierung angeführt. Die Liste der Beispiele erhebt bei Weitem keinen Anspruch auf Vollständigkeit - vieles mehr hätte hinzugefügt werden können. Im Anschluss daran bieten wir einige Illustrationen für die verschiedenen Möglichkeiten einer Neudefinition der Untersuchungseinheit im methodologischen Kosmopolitismus. 
Tabelle: Untersuchungseinheiten im methodologischen Kosmopolitismus

\begin{tabular}{|c|c|c|}
\hline & Prozesse & Strukturen \\
\hline Einbettung des Nationalen & $\begin{array}{l}\text { „compressed modernization” } \\
\text { (Chang 1999, } 2010 \text { a,b); } \\
\text { „kosmopolitische Europäisie- } \\
\text { rung“ (Beck / Grande 2004); } \\
\text { „recursive cosmopolitization“ } \\
\text { (Levy 2010) }\end{array}$ & $\begin{array}{l}\text {,transnationale Politikre- } \\
\text { gime“ (Grande 2004); } \\
\text { "global assemblages“" } \\
\text { (Sassen 2008) } \\
\text { world regions“" } \\
\text { (Katzenstein 2005) }\end{array}$ \\
\hline Ersetzung des Nationalen & $\begin{array}{l}\text { "ships in motion” (Gilroy 1993); } \\
\text { "supply chain capitalism” (Tsing } \\
\text { 2009); } \\
\text { "cosmopolitan innovations" (Ty- } \\
\text { field / Urry 2010) }\end{array}$ & $\begin{array}{l}\text { „,world religions“; } \\
\text { „,transnational migrant net- } \\
\text { works“(Glick Schiller 2009); } \\
\text { „,border zones; ,"global city“; } \\
\text {,the local“; ,the family“; “the } \\
\text { individual”" }\end{array}$ \\
\hline
\end{tabular}

a) Ships in motion: die Ersetzung des Nationalen durch transnationale Prozesse. Eine der ersten und radikalsten Alternativen zum methodologischen Nationalismus stammt (wenn auch dort nicht als solche beschrieben) von Paul Gilroy. In seiner wegweisenden Untersuchung nahm er sich nicht den nationalen „Container”, sondern einen transkontinentalen Raum, den Black Atlantic (1993), als Feld seiner Theoriebildung und empirischen Forschung. Überzeugend argumentierte er, dass die Vorstellungen von Nation, Nationalität und nationaler Zugehörigkeit weitreichende epistemologische Folgen haben: Sie bestätigen ein Forschungsprogramm und praktizieren ein „kulturelles Insidertum” auf Grundlage einer Essentialisierung ethnischer Unterschiede. Gilroys Ausgangspunkt ist daher keine feste Einheit; vielmehr ist das Bild von ships in motion zwischen Europa, Amerika, Afrika und der Karibik das zentrale symbolische Konzept und Organisationsprinzip seiner Forschung. ,The image of the ship - a living, micro-cultural, micro-political system in motion - is especially important for historical and theoretical reasons. [...] Ships immediately focus attention on the middle passage, on the various projects for redemptive return to an African homeland, on the circulation of ideas and activists as well as the movement of key cultural artefacts: tracts, books, gramophone records, and choirs." (Gilroy 2008: 204)

Eine ähnliche Herangehensweise der Ersetzung des Nationalen als Basiseinheit der Forschung durch neue transnationale Prozesse wählte Anna Tsing (2009) in ihrer Studie über neue Formen eines supply chain capitalism. Im Gegensatz zu Theorien zunehmender kapitalistischer Konvergenz und Homogenität stellt ihre Theorie die fundamentale Rolle der Differenz bei der Mobilisierung von Kapital, Arbeit und anderen Ressourcen heraus. Der Fokus und Untersuchungsgegenstand in ihrer Forschung ist wiederum keine feste Einheit, sondern ein Prozess: die Mobilisierung von Arbeit in Versorgungsketten in ihrer Abhängigkeit von Geschlecht, Ethnizität, Nationalität, Religion und Bürgerstatus. Ihre Analyse zeigt, dass Vielfalt für den globalen Kapitalismus nicht nur dekorativ, sondern von zentraler Bedeutung ist. Ein weiteres Beispiel sind die „,cosmopolitan innovations” (Tyfield / Urry 2010 in diesem Heft), ein transnationaler Prozess, der die internationale Zusammenarbeit bei Innovationen stimuliert, die für die Entwicklung verschiedener ,globaler Güter” nötig sind.

b) Transnationale Netzwerke, Grenzgebiete und Imperien: Die Ersetzung des Nationalen durch neue transnationale Strukturen. Das vergangene Jahrzehnt brachte in erheblichem Umfang Studien im Feld transnationaler Forschung hervor (Khagram / Levitt 2008; Pries 2008 a,b). Diese Studien weisen ungeachtet der verschiedenen Forschungsziele und akademischen Disziplinen die Gemeinsamkeit auf, dass sie das Nationale als Untersuchungseinheit durch neue Strukturen ersetzen, die auf verschiedenen Ebenen beobachtet werden können. Ein höchst innovativer Ansatz wurde hier in der Forschung von Nina Glick Schiller (2009) und 
anderen gewählt (siehe Khagram / Levitt 2008; Vertovec 2009). Sie stützen sich auf eine konstruktivistische Sicht territorialer Räume, in denen transnationale soziale Formationen wie transnationale Netzwerke, Verwandtschaftsgruppen, Migrantenorganisationen und Diaspora-Gemeinschaften - die Territorien der Nationalstaaten kreuzen und sich mit ihnen überschneiden, ohne unbedingt von globaler Reichweite zu sein. Ein anderes Beispiel wären die verschiedenen Konzepte des Imperiums, die in den vergangenen Jahren im Zusammenhang mit der Rolle der USA bei der Neugestaltung einer globalen Weltordnung und in Bezug auf den europäischen Integrationsprozess entwickelt wurden (vgl. Beck / Grande 2004: Kap. III; Katzenstein 2005).

c) Compressed modernization, globale Netzwerke, transnationale Politikregime: Einbettung des Nationalen in neue transnationale Strukturen und Prozesse. Die Kosmopolitisierung moderner Gesellschaften bedeutet jedoch nicht zwangsläufig das Ende des Nationalen. Alle Voraussagen über das Ende des Nationalstaats im Zeitalter der Globalisierung haben sich bislang als verfrüht erwiesen. Der Nationalstaat löst sich im Prozess der reflexiven Modernisierung nicht auf; er transformiert sich vielmehr in verschiedenster Weise (Sørensen 2004; Grande / Pauly 2005; Sassen 2008; Grande 2008). Dies impliziert, dass das Nationale, also nationale Grenzen, nationale Monopole auf den legitimen Gewaltgebrauch, nationale Normen, Institutionen und Kulturen nach wie vor mächtige, gesellschaftsprägende Faktoren sein können. Deshalb kann ein methodologischer Kosmopolitismus das Nationale nicht völlig vernachlässigen.

Die Einbettung des Nationalen in Prozesse der Kosmopolitisierung kann auf sehr unterschiedliche Weise geschehen. Entsprechend vielfältig sind die neuen Untersuchungseinheiten, die in dieser Variante des methodologischen Kosmopolitismus entwickelt wurden. Ein erstes Beispiel ist das Konzept der ,transnationalen Politikregime” (Grande 2004; Grande / Pauly 2005; Grande et al. 2007). Es bezieht sich auf neue Formen der transnationalen Institutionenbildung, die sich im Zusammenhang mit einer Reihe globaler Regelungsprobleme wie dem Klimawandel, dem Internet oder der Besteuerung transnationaler Unternehmen herausgebildet haben. Diese Institutionen organisieren transnationale Interaktionen, deren Grenzen nicht durch nationale Hoheitsrechte definiert werden, sondern durch ein spezifisches Regulationsproblem; sie integrieren sehr verschiedene und extrem variable Gruppen von Akteuren (öffentliche und private) und sie erstrecken sich über verschiedene territoriale Ebenen. Für eine empirische Analyse transnationaler Politik sind diese Politikregime vielfach die angemessenste Untersuchungseinheit. Entscheidend ist hier, dass diese neuen Institutionen den Nationalstaat nicht ersetzen, sondern ihn vielmehr integrieren. Die Nationalstaaten sind in neue transnationale Regulationssysteme eingebettet, und eine der wichtigsten Aufgaben empirischer Forschung ist die Untersuchung der spezifischen Bedeutung, die sie im Rahmen dieser Institutionen annehmen. Wo der Nationalstaat seine Dominanz fortsetzt, wie wir es gegenwärtig in der Klimapolitik beobachten können, dort droht die transnationale Ebene zu einem bloßen „Schauplatz“ des Nationalen zu verkümmern.

Ein weiteres Beispiel dafür, warum es nötig sein kann, den Nationalstaat weiterhin als Untersuchungseinheit heranzuziehen und dennoch die Enge des methodologischen Nationalismus zu überwinden, findet man in den Arbeiten von Daniel Levy (2010) und Saskia Sassen (2008). Diese zeigen, dass Globalisierungsprozesse von Transformationen innerhalb von Nationalstaaten abstammen, von denen sie auch ihre Dynamik gewinnen, ob es sich um den Aufstieg des globalen Kapitalmarktes handelt oder um Netzwerke von Menschenrechtsaktivisten (Kurasawa 2007). Kurz: Der nationale und lokale Raum wird hier zu einem hochkomplexen „Schauplatz des Globalen“. In diesem Fall gewinnen die nationalen Institutionen und Kulturen, ihre Pfadabhängigkeit und jeweilige Geschichte eine neue - und nicht unbedingt geringere Bedeutung. Gerade wegen der Verwobenheit der Welt beginnt vieles lokal, wenn auch oft 
„translokal“. Da das grenzüberspannende Netz strategisch handelnder subnationaler Akteure, das aus intensiven Transaktionen, Experten- und Informationsströmen geknüpft ist, eine Verkörperung denationalisierter Räumlichkeit und Zeitlichkeit darstellt, ist es weder national noch global, sondern kosmopolitisch.

Die transnationalen Räume, Prozesse und Strukturen, die in der kosmopolitischen Methodologie die Untersuchungseinheit bilden, können in verschiedener Weise konstruiert werden. Sie können entweder deduktiv aus theoretischen Grundbegriffen abgeleitet werden, wie wir es beispielhaft für die Fälle von Individualisierung und Risiko gezeigt haben, oder aber induktiv auf der Grundlage von (1) historischen, (2) funktionalen, (3) sozialen oder (4) institutionellen Kriterien identifiziert werden (Grande 2006). Empirische Studien im Münchener Forschungsprogramm zur reflexiven Modernisierung haben Beispiele für beide dieser Möglichkeiten zutage gefördert (vgl. insbesondere Beck / Bonß 2001; Beck / Lau 2004; Bonß / Lau 2010).

Die Möglichkeit historisch definierter Untersuchungseinheiten wird repräsentiert durch die „transnationalen Erinnerungsräume”, die Daniel Levy und Natan Sznaider in ihrer Arbeit über den Holocaust identifizieren (Levy / Sznaider 2007). Diese transnationalen Erinnerungsräume sind durch gemeinsame historische Erfahrungen entstanden, die der Holocaust konstituiert. Obwohl die mitteleuropäischen Länder, die am direktesten davon betroffen waren, verschiedene Praxen und Politiken des Umgangs mit dem Holocaust hervorgebracht haben, können alle diese sozialen Aktivitäten nur adäquat verstanden werden, wenn man sie im Kontext der „transnationalen Erinnerungsräume” interpretiert, in die sie eingebettet sind.

Eine zweite Möglichkeit sind funktional definierte Untersuchungseinheiten, wie sie uns im Fall der bereits erwähnten ,transnationalen Politikregime” begegnen. Hier sind der thematische Kern und die Grenzen der Untersuchungseinheit durch gemeinsame Regulierungsprobleme konstituiert, etwa der Finanzmärkte, der Unternehmensbesteuerung oder des Klimaschutzes (vgl. z.B. Drezner 2007). Hiermit soll jedoch keine funktionalistische Sicht sozialer Probleme nahegelegt werden. Es gibt eine Fülle empirischer Untersuchungen, die zeigen, dass die behandelten Probleme selbst in politischen Konflikten mit unterschiedlichen Arten und Graden der Politisierung konstruiert werden, und dasselbe gilt für den institutionellen Rahmen, der zu ihrer Bewältigung geschaffen wurde. Da die Konflikte sich aber auf spezifische Regulationsprobleme konzentrieren, ist es dennoch sinnvoll, diese Probleme als Ausgangspunkte und Bezugsrahmen empirischer Analysen heranzuziehen.

Drittens können wir transnationale Räume und Untersuchungseinheiten identifizieren, indem wir an sozialen Praktiken, insbesondere sozialen Konflikten und Konfliktstrukturen, ansetzen. Beispiele dafür sind „transnationale politische Kampagnen” und „Debatten” (vgl. Kriesi et al. 2010). Während Kampagnen durch politische Ereignisse und Akteure konstituiert werden, definieren sich Debatten durch ihren jeweiligen thematischen Kern, z.B. Immigration und europäische Integration. Die Analyse transnationaler Prozesse mit Hilfe dieser Konzepte ermöglicht es, sich empirisch auf zwei Schlüsselvariablen zu konzentrieren: Variationen der Akteurskonstellationen (z.B. politische Parteien, transnationale Organisationen usw.) und Variationen in der territorialen Reichweite politischer Aktivitäten (z.B. national, europäisch). Ein solcher analytischer Ansatz eröffnet die Möglichkeit, verschiedene Formen politischer Mobilisierung und Artikulation auf einen gemeinsamen Begriff zu bringen und die ihnen inhärente Struktur politischer Konflikte grenzüberschreitend zu untersuchen.

Schließlich können transnationale Untersuchungseinheiten auch institutionell, anhand neuer Formen der transnationalen Institutionenbildung, identifiziert werden. In diesem Fall, für den sich insbesondere in der Europaforschung Beispiele finden (Beck / Grande 2004; Delanty / Rumford 2005), ist es der Prozess formaler und informaler regionaler Integration mit seinen Folgen, der den Bezugsrahmen für Gesellschaftstheorie und empirische Analyse bietet. Wie 
Peter Katzenstein (2005) in seiner Arbeit über die „Welt der Regionen” gezeigt hat, eröffnet dies zugleich neue Möglichkeiten für vergleichende Forschung zwischen den Weltregionen.

\section{Perspektiven kosmopolitischer Soziologie: Normative Fragen, Adressaten, Möglichkeiten der Verwirklichung}

Die kosmopolitische Wende in Theorie und empirischer Forschung wäre unvollständig, wenn man den methodologischen Kosmopolitismus nur konzeptuell verstünde und der Verschränkung von epistemologischem Objekt und Verwendungskontext, die allgemein charakteristisch für die Sozialwissenschaften ist, kein zentraler Stellenwert zukäme. Dies wirft drei Fragen auf: (1) die normative Frage: Was sind die kosmopolitischen Alternativen und Visionen? Und unter welchen Bedingungen führt ,Kosmopolitisierung “ $z u$,,reflexiven Kosmopolitismen“ und nicht zu „reflexiven Fundamentalismen“"? (2) Wer sind die Adressaten kosmopolitischer Sozialwissenschaften? (3) Wie realistisch sind kosmopolitische Ideen und Alternativen angesichts der Dominanz des Nationalen und der Re-Ethnisierungstendenzen überall auf der Welt sowie der Pluralität von Risikowahrnehmungen und Antagonismen? Muss der Kosmopolitismus eine idealistische Utopie bleiben?

\section{Normative Probleme}

„Ich spreche zu Ihnen nicht als Präsidentschaftskandidat, sondern als Bürger, als stolzer Bürger der Vereinigten Staaten und Weltbürger", verkündete Barack Obama vor einer Menge jubelnder Berliner. Seit Immanuel Kant herrscht kein Mangel an normativen Ideen und Vorschlägen bezüglich der Frage, was „Weltbürger“ bedeutet. Die Verbindung zwischen Kosmopolitismus, Patriotismus und Nationalismus hat bereits die größten und kreativsten Denker im Europa des 18. und frühen 19. Jahrhunderts beschäftigt (Delanty 2009: Kap. 1). Im 20. Jahrhundert dagegen ist der Kosmopolitismus als ernstzunehmende intellektuelle und politische Position zunächst fast verschwunden. Erst nach dem Zusammenbruch des Sowjetkommunismus haben die Kantischen Träume vom ,ewigen Frieden“ abermals die öffentliche Vorstellungskraft beflügelt und wieder eine überraschende Aktualität in verschiedenen Forschungsfeldern gewonnen. Die ausschlaggebende Frage ist hier jedoch: Wie kosmopolitisch ist die normative Debatte über Kosmopolitismus oder besser: Kosmopolitismen? Gibt es zum Beispiel feministische Visionen des Kosmopolitismus, die nicht auf dem männlichen Maximalismus des „Weltbürgers“ basieren? „Ein jeder Kosmofeminismus müsste einen kritisch engagierten Raum schaffen, der nicht nur eine Leinwand der Globalisierung oder ein Gegenmittel zum Nationalismus ist, sondern ein Fokus auf Projekten der Intimsphäre, verstanden als Teil des Kosmopolitischen” (Pollock et al. 2000: 584). Vor allem stellt sich die Frage: „Wie radikal können wir die Geschichte des Kosmopolitismus neu schreiben und wie dramatisch ihn neu kartieren, wenn wir bereit sind, außerhalb der Schublade europäischer Geistesgeschichte zu denken" (ebd.: 586)? Unser Ansatz zur Konzeptualisierung dieser normativen Frage sieht die Unterscheidung dreier Zukunftsszenarien vor - eines optimistischen, eines realistischen und eines pessimistischen.

Das optimistische Szenario geht von einer verdeckten Verbindung zwischen dem Klimawandel und Immanuel Kant aus. Es erfordert in der Tat einen entschiedenen Schritt, das Zurücklegen zumindest eines Teils des Weges zum ,ewigen Frieden”, um eine wirksame Antwort auf den Klimawandel zu finden. Das heißt, in der Formel wissenschaftlicher und ökonomischer Rede von Weltrisiken ist etwas Normatives, Großartiges, geradezu Undenkbares verborgen, dessen Realismus Teil des Realismus ist, der im Zusammenhang mit der drohenden ökologischen Apokalypse an Bedeutung gewinnt. Es genügt nicht (um Max Webers berühmte Unterscheidung zu übernehmen), nur gut sein zu wollen - einer kosmopolitischen Gesinnungsethik anzuhängen. Eine kosmopolitische Verantwortungsethik ist ebenfalls vonnöten - oder, um noch ambitionierter zu formulieren: Was benötigt wird, ist die Konstitution eines politi- 
schen Subjekts namens „Menschheit”. Der neue normative Imperativ lautet: Individuen, Neoliberale und Neonationalisten, Konzerne, soziale Gruppierungen und Gesellschaften, Mächtige und Schwache der Welt - nur wenn ihr euch gemeinsam an einen Tisch setzt und die Bedingungen eines globalen Deals über eine gerechte Balance verhandelt - also ein wenig Kant in der Welt verwirklicht -, habt ihr eine Chance zu überleben. Dieser teuflische Pakt einer Zivilisation, die sich selbst zu zerstören droht - die keineswegs nur normative Gnadenlosigkeit der Überlebensmaxime: Kant oder Katastrophe! Kooperieren oder scheitern! - gibt vielleicht Grund zur Hoffnung.

Das realistische Szenario sieht wie folgt aus: Es ist der Antagonismus zwischen Produzenten und Empfängern von Klimarisiken, der eine Kosmopolitik des Klimawandels zugleich konstituiert und blockiert. Es gibt keine Lösung für dieses Dilemma. Deshalb werden die Agnostiker des Klimawandels den längeren Atem haben; eine Art von öko-technologischer Modernisierung wird in Richtung eines mehr oder weniger ,grünen Kapitalismus” voranschreiten; je mehr die Hauptströmungen in den politischen Parteien sich darüber einig sind, den großen Tanker weiter geradeaus fahren zu lassen, umso absurder und unglaubwürdiger werden ihre inszenierten Differenzen erscheinen. Dies entspricht der klassischen Position, die Niklas Luhmann (1984) vertrat: Für das Überleben genügt Evolution - fundamentale Änderung geht sowieso nicht. Es bleibt nur die zynische Maxime: Aufregungsschäden minimieren! Schweigen entgiftet!

Das pessimistische Szenario lässt die Mainstream-Position durchaus nicht außer Acht, vielleicht folgt es sogar aus ihr: Die radikal ungleichen Auswirkungen des Klimawandels und der Klimapolitik selbst werden zu Öl im Feuer fundamentalistischer Gegenbewegungen. Anders ausgedrückt: Ein tödlicher Teufelskreis von Klimakatastrophen, Migrationsströmen, fundamentalistischem Nationalismus und religiösem Fundamentalismus kommt in Gang und führt zum Ausbruch von Gewalt bis hin zu Klimakriegen. ${ }^{9}$

Natürlich werden sich diese Szenarien in verschiedenen Modernisierungskontexten unterschiedlich ausgestalten bzw. sich anders ineinander fügen. Vor diesem Hintergrund kann das normative Problem genauer in sozialwissenschaftlichen Begriffen formuliert werden: Erstens, unter welchen Bedingungen nimmt die „Dialektik“ der kosmopolitischen Modernen die Form des positiven Szenarios an, eröffnet also praktische Handlungsoptionen zur institutionellen Festigung kosmopolitischer Gemeinschaften globaler Risiken? Dies schließt zweitens die Frage ein, welche Akteure die Hegemonialkonflikte dominieren werden, zu denen es dabei kommen kann, wohl auch wird, und welche Machtstrategien ihnen zur Verfügung stehen werden (Beck 2002). Normativer Kosmopolitismus ist jedoch nicht nur das Produkt internationaler und innernationaler Konflikte, sondern erfordert auch deren kosmopolitische Zivilisierung. Damit stellt sich drittens die Frage, wie eine ökologische und soziale Zivilisierung des Kapitalismus jenseits post-souveräner Nationalstaaten und eines utopischen Weltstaats möglich sein kann? Diese Fragen eröffnen theoretische, normative und politische Problemhorizonte, in denen kosmopolitische Sozialwissenschaften eine kreative, politisch höchst relevante Aufgabe finden können. Diese normative Wende in der kosmopolitischen Gesellschaftstheorie und -forschung gerät unausweichlich in Opposition sowohl zum zeitgenössischen Geist des neoliberalen Kapitalismus wie zum a priori des Nationalstaats. Genau dies wäre ihr aufklärerischer Stachel.

9 Empirische Analysen der politischen Folgen der Globalisierung in Westeuropa haben gezeigt, dass die Globalisierung eine neue Konfliktlinie (,cleavage“) zwischen Kosmopolitismus und Nationalismus geschaffen hat, die bislang am erfolgreichsten von neuen rechtspopulistischen Parteien artikuliert wurde, die eine Renationalisierung der Politik im Zeitalter der Globalisierung anstreben (Kriesi et al. 2008). 


\section{Wer sind die Adressaten kosmopolitischer Sozialwissenschaften?}

Bereits der methodologische Nationalismus schließt implizit Annahmen ein, die kategorische Rahmen in eine praktisch-politische Beziehung zu den Adressaten der jeweiligen Theorien setzen. Im „Container-Modell” wird die Definition der nationalstaatlichen Gesellschaft als Forschungsgegenstand mit mehr oder weniger exklusiven Bezügen auf nationale Akteure (Regierungen, politische Parteien, Gewerkschaften, Klassen, Eliten, soziale Bewegungen usw.) kurzgeschlossen. Diese dienen als Hauptobjekte der Theoriebildung und empirischen Forschung (sogar unbeabsichtigt) und als Adressaten praktischer Empfehlungen und Interventionen. Wir können hier zwei Arten von Wertbeziehungen (Max Weber) und Parteilichkeiten unterschieden: explizite und implizite. Die Werte und Parteilichkeiten, die sich explizit im methodologischen Nationalismus bündeln, konzentrierten sich nach dem Zweiten Weltkrieg im europäischen Kontext auf den sich entwickelnden demokratischen, interventionistischen Wohlfahrtsstaat als neuen Repräsentanten des Gemeinwohls und -willens. Der Siegeszug von Soziologie und Politikwissenschaft nach dem Zweiten Weltkrieg in Westeuropa war eng verknüpft mit der Doppelaufgabe, zum einen den Rückfall in den Faschismus und Nationalsozialismus und zum anderen das Überlaufen der Arbeiterklasse zur kommunistischen Konkurrenz zu verhindern. Darüber hinaus kann leicht im Detail gezeigt werden (wenn auch hier nicht der Raum dafür ist), dass die heute dominante akademische Selbstbeschreibung, dass nämlich Gesellschaftstheorie und -forschung ,wertfrei” und zu ,rein wissenschaftlichen Zwecken” betrieben werde, in klarem Widerspruch zu implizit existierenden Parteilichkeiten steht. Dies sind die Folgen der unreflektierten Übernahme der nationalen „Akteursperspektive” in die „Beobachterperspektive” in den Sozialwissenschaften, zum Beispiel durch den Ausschluss der Externalisierung von Risiken aus dem sozialwissenschaftlichen Forschungshorizont. Im Fall der neuen globalen Risiken ist Wertfreiheit kein Zeichen von Reife, sondern schlicht ein Selbstmissverständnis. Überdies bleibt die Schlüsselfrage nach der Verantwortung der Sozialwissenschaften in der sich selbstgefährdenden Zivilisation, die die Basisprinzipien der Moderne entzaubert, ungestellt.

Im Zeitalter der Globalisierung ist der methodologische Nationalismus nicht nur als begrifflicher Rahmen, sondern auch in seinem Adressatenbezug antiquiert. Dies ist ein wesentlicher Grund dafür, dass sich die hoch professionalisierte Sozialwissenschaft einerseits in einem Nirvana der Abstraktionen verliert und zugleich in eine esoterische Fragmentierung unverbundener, hoch spezialisierter empirischer Forschungsprojekte zerfällt (Burawoy 2005). Mit anderen Worten: Eine deprovinzialisierte und relokalisierte, post-universalistische Gesellschaftstheorie, die ihre europäischen Wurzeln wiederentdeckt und reflektiert, befindet sich auf der Suche nach einer neuen, historischen „Wertbeziehung”. Diese neue Wertbeziehung müsste über kulturelle (Zukunfts-)Geltung verfügen und die Wiederentdeckung der Geschichtlichkeit von Gesellschaft und Moderne verbinden mit der Suche nach neuen Adressaten.

Wie wir sahen, fällt im methodologischen Nationalismus der aus nationalstaatlichen Kategorien gebildete Forschungsrahmen (sozusagen hinter dem Rücken des Forschers) mit spezifisch nationalen Akteurskategorien (etwa sozialen Klassen, Bewegungen der Zivilgesellschaft, nationalen Regierungen, Gewerkschaften usw.) zusammen. Dies gilt nicht für den Adressatenbezug kosmopolitischer Sozialwissenschaft. Im Gegenteil, „kosmopolitische Wende” bedeutet im Hinblick auf den Adressatenbezug: keine spezifischen Akteurskategorien, also weder Regierungen noch Gewerkschaften, Wirtschaftsunternehmen oder -verbände, soziale Klassen oder soziale Bewegungen. Vor allem folgt eine kosmopolitische Sozialwissenschaft keiner falschen Analogie, indem sie nach neuen ,transnationalen Klassen”, ,, Multitudes”, oder „Eliten" sucht, um sich mit ihnen zu vereinen. Vielmehr sind die Adressaten kosmopolitischer Gesellschaftstheorie und -forschung kosmopolitische Koalitionen von Akteuren in all ihrer Vielfalt. In eben diesen heterogenen, ständig fluktuierenden Koalitionen, zu denen Regierun- 
gen ebenso gehören können wie nationale und internationale „Sub-Politiken”, Konzerne, ,glokale" zivilgesellschaftliche Gruppen, internationale Organisationen, informelle Versammlungen von Staaten (wie G8 und G20) usw., werden die Kämpfe zwischen konfligierenden hegemonialen Projekten ausgetragen, die den Anspruch erheben, das Universelle zu repräsentieren und die symbolischen Parameter des sozialen Lebens zu definieren. Und indem über alle Arten von Grenzen hinweg solche Koalitionen geschmiedet werden, öffnen sich unter Bedingungen, die noch der Erforschung harren, neue Sphären und Räume politischen Handelns. Mit einer neuen Qualität globaler Abhängigkeiten und Interdependenzen konfrontiert kann kein einzelner Akteur allein erwarten zu gewinnen; sie alle bedürfen der Koalitionen, Allianzen und Netzwerke (Beck 2002). Dies ist der Weg, auf dem das vom Nebel verhüllte Machtspiel der „Weltinnenpolitik" seine eigenen immanenten Alternativen und Oppositionen eröffnet und neuartige kollektive Identitäten und politische Subjekte hervorbringt.

\section{Möglichkeiten der Verwirklichung}

Bis hierher ist unsere Diagnose zwiespältig, Kritiker könnten sagen: widersprüchlich; manche Theoretiker halten für solche Fälle das Allzweckwort ,dialektisch” bereit. Dies wirft die letzte Frage auf: Wie können globale Risiken unter den Bedingungen multipler, miteinander wetteifernder Modernen mit ihren verschiedenen normativen Modellen, materiellen Interessen und politischen Machtkonstellationen erfolgreich bearbeitet werden? Den Schlüssel zur Antwort bildet das Konzept der kosmopolitischen Realpolitik. Um dieses Konzept zu verstehen und zu entwickeln, muss es insbesondere vom normativ-philosophischen Kosmopolitismus auf der einen und vom idealistisch-utopischen (Held 2007; Archibugi 2008) auf der anderen unterschieden werden. Kosmopolitische Realpolitik appelliert nicht (jedenfalls nicht vorrangig) an geteilte Ideen und Identitäten, sondern fordert, Macht und Interessen ins Spiel zu bringen. Wenn wir eine solche ,realistische” Perspektive einnehmen, ist die entscheidende Frage, wie das hegemoniale „Meta-Machtspiel” der Weltinnenpolitik gestaltet und in seinem Rahmen Interessen verfolgt werden können, so dass sie der Verwirklichung gemeinsamer kosmopolitischer Ziele dienen? Kurz (und Mandeville folgend): Wie können private Untugenden in öffentlichen, kosmopolitischen Nutzen umgewandelt werden?

Das Konzept der kosmopolitischen Realpolitik, das sich die Beantwortung dieser Frage zum Ziel setzt, beruht auf vier Annahmen. Erstens: In der neuen historischen Realität der Weltrisikogesellschaft kann keine Nation ihre Probleme allein bewältigen. Wer die nationale Karte spielt, verliert. Zweitens: Globale Probleme bringen neue kosmopolitische Imperative mit sich, die wiederum transnationale Risikogemeinschaften entstehen lassen. Drittens: Internationale Organisationen sind nicht bloß die Fortsetzung nationaler Politik mit anderen Mitteln. Sie können nationale Interessen transformieren. Viertens: Kosmopolitischer Realismus ist zugleich ökonomischer Realismus. Er senkt Kosten und verteilt sie um, denn mit dem Verlust von Legitimität steigen Kosten exponentiell.

So verstanden impliziert der Kosmopolitismus einen spezifischen Ansatz, um sicherzustellen, dass die eigenen (individuellen oder kollektiven) Interessen vorankommen und Geltung besitzen. Der kosmopolitische Realismus fordert weder die Opferung von Eigeninteressen noch eine exklusive Privilegierung höherer Ideen und Ideale. Er anerkennt im Gegenteil, dass politisches Handeln größtenteils interessengeleitet ist. Er beharrt jedoch auf einer ganz bestimmten Art, Eigeninteressen zu verfolgen, so dass diese mit denen einer größeren Gemeinschaft kompatibel sind. Kosmopolitischer Realismus bedeutet daher die Anerkennung der legitimen Interessen anderer und ihre Einbeziehung in das eigene Kalkül. In diesem Prozess werden Interessen durch wiederholte, gemeinsame Strategien der Selbstbeschränkung zu ,reflexiven Nationalinteressen"; genauer gesagt: Ermächtigung oder Empowerment erwächst aus Selbstbeschränkung. Ideal wäre es, wenn nationale und globale, individuelle und kollektive 
Ziele zugleich erreicht werden könnten. In Wirklichkeit jedoch stößt die kosmopolitische Realpolitik oft auf Grenzen und Dilemmata. Sie ist kein Allheilmittel für die Probleme der Welt und funktioniert bei Weitem nicht immer. Es hängt insbesondere vom normativen und institutionellen Rahmen ab, in dem Entscheidungen zu treffen sind, ob eine kosmopolitische Lösung des jeweiligen Problems möglich ist. ${ }^{10}$ Dennoch ist die Hauptbotschaft kosmopolitischer Realpolitik: Die Zukunft ist offen. Sie hängt von unseren Entscheidungen ab. Unsere Forschung und die Begriffe und Konzepte, die wir verwenden, haben eine Wirkung.

\section{Literatur}

Albrow, Martin (2007): Das globale Zeitalter, Frankfurt / Main.

Anderson, Benedict (1996): Die Erfindung der Nation. Zur Karriere eines folgenreichen Konzepts, Frankfurt / Main.

Appadurai, Arjun (1996): Modernity at Large: Cultural Dimensions of Globalization, Minneapolis.

Appadurai, Arjun (1998): Globale ethnische Räume. Bemerkungen und Fragen zur Entwicklung einer transnationalen Anthropologie, in: Ulrich Beck (Hrsg.), Perspektiven der Weltgesellschaft, Frankfurt / Main, S. 11-40.

Appiah, Kwame Anthony (2007): Der Kosmopolit. Philosophie des Weltbürgertums, München.

Archibugi, Daniele (2008): The Global Commonwealth of Citizens: Towards Cosmopolitan Democracy, Princeton.

Arnason, Johann P. (2003): Civilizations in Dispute: Historical Questions and Theoretical Traditions, Leiden.

Arnoldi, Jakob (2009 a): Risk: An Introduction, Cambridge.

Arnoldi, Jakob (2009b): Alles Geld verdampft. Finanzkrise in der Weltrisikogesellschaft, Frankfurt / Main.

Baban, Feyzi (2006): Living with Difference: Cosmopolitanism, Modernity, and Political Community, in: Studies in Political Economy 77, S. 105-126.

Bauman, Zygmunt (2001): The Individualized Society, Cambridge.

Bayly, Christopher A. (2006): Die Geburt der modernen Welt. Eine Globalgeschichte 1780-1914, Frankfurt / Main.

Beck, Ulrich (1983): Jenseits von Stand und Klasse? Soziale Ungleichheiten, gesellschaftliche Individualisierungsprozesse und die Entstehung neuer sozialer Formationen und Identitäten, in: Reinhard Kreckel (Hrsg.), Soziale Ungleichheiten (Sonderband 2: Soziale Welt), Göttingen, S. 35-74.

Beck, Ulrich (1986): Risikogesellschaft. Auf dem Weg in eine andere Moderne, Frankfurt / Main.

Beck, Ulrich (1993): Die Erfindung des Politischen. Zu einer Theorie reflexiver Modernisierung, Frankfurt / Main.

Beck, Ulrich (1996): Weltrisikogesellschaft, Weltöffentlichkeit und globale Subpolitik. Ökologische Fragen im Bezugsrahmen fabrizierter Unsicherheiten, in: Andreas Diekmann / Carlo C. Jaeger (Hrsg.), Umweltsoziologie (Sonderheft 36: Kölner Zeitschrift für Soziologie und Sozialpsychologie), Opladen, S. 119-147.

Beck, Ulrich (1997): Was ist Globalisierung? Irrtümer des Globalismus - Antworten auf Globalisierung, Frankfurt / Main.

Beck, Ulrich (1999): World Risk Society, Cambridge.

Beck, Ulrich (2002): Macht und Gegenmacht im globalen Zeitalter. Neue weltpolitische Ökonomie, Frankfurt / Main.

10 In unserem Buch „Das kosmopolitische Europa“ haben wir einige dieser Dilemmata kosmopolitischer Realpolitik beschrieben (vgl. Beck / Grande 2004: Kap. 8). 
Beck, Ulrich (2004): Der kosmopolitische Blick oder: Krieg ist Frieden, Frankfurt / Main.

Beck, Ulrich (2007): Weltrisikogesellschaft. Auf der Suche nach der verlorenen Sicherheit, Frankfurt / Main.

Beck, Ulrich / Elisabeth Beck-Gernsheim (Hrsg.) (1994): Riskante Freiheiten. Individualisierung in modernen Gesellschaften, Frankfurt / Main.

Beck, Ulrich / Elisabeth Beck-Gernsheim (2001): Individualization: Institutionalized Individualism and its Social and Political Consequences, London.

Beck, Ulrich / Elisabeth Beck-Gernsheim (2011): Das globale Chaos der Liebe, Berlin (im Erscheinen).

Beck, Ulrich / Wolfgang Bonß (Hrsg.) (2001): Modernisierung der Moderne, Frankfurt / Main.

Beck, Ulrich / Wolfgang Bonß / Christoph Lau (2001): Theorie reflexiver Modernisierung - Fragestellungen, Hypothesen, Forschungsprogramm, in: Ulrich Beck / Wolfgang Bonß (Hrsg.), Die Modernisierung der Moderne, Frankfurt / Main, S. 11-59.

Beck, Ulrich / Anthony Giddens / Scott Lash (1996): Reflexive Modernisierung. Eine Kontroverse, Frankfurt / Main.

Beck, Ulrich / Edgar Grande (2004): Das kosmopolitische Europa. Gesellschaft und Politik in der Zweiten Moderne, Frankfurt / Main.

Beck, Ulrich / Edgar Grande (Hrsg.) (2010): Special Issue: Varieties of Second Modernity: Extra-European and European Experiences and Perspectives, British Journal of Sociology 61 / 3.

Beck, Ulrich / Christoph Lau (Hrsg.) (2004): Entgrenzung und Entscheidung. Was ist neu an der Theorie reflexiver Modernisierung?, Frankfurt / Main.

Beck, Ulrich / Christoph Lau (2005): Theorie und Empirie reflexiver Modernisierung. Von der Notwendigkeit und den Schwierigkeiten, einen historischen Gesellschaftswandel innerhalb der Moderne zu beobachten und zu begreifen, in: Soziale Welt 56, S. 107-135.

Beck, Ulrich / Angelika Poferl (Hrsg.) (2010): Große Armut, großer Reichtum: Transnationalisierung sozialer Ungleichheiten, Berlin.

Beck, Ulrich / Natan Sznaider (Hrsg.) (2006 a): Special Issue on Cosmopolitan Sociology: British Journal of Sociology 57 / 1.

Beck, Ulrich / Natan Sznaider (2006 b): Unpacking Cosmopolitanism for the Social Sciences: A Research Agenda, in: British Journal of Sociology 57, S. 1-23.

Beck, Ulrich / Natan Sznaider (2006 c): A Literature on Cosmopolitanism: An Overview, in: British Journal of Sociology 57, S. 153-164.

Beck, Ulrich / Natan Sznaider (2010): New Cosmopolitanism in the Social Sciences, in: Bryan S. Turner (Hrsg.), The Routledge International Handbook of Globalization Studies, Abingdon / Oxon - New York, S. 635-652.

Beck-Gernsheim, Elisabeth (1983): Vom „Dasein für andere“ zum Anspruch auf ein Stück „,eigenes Leben“ - Individualisierungsprozesse im weiblichen Lebenszusammenhang, in: Soziale Welt 34, S. 307-341.

Beitz, Charles R. (2005): Cosmopolitanism and Global Justice, in: Journal of Ethics 9, S. 11-27.

Benhabib, Seyla (2007): Twilight of Sovereignty or the Emergence of Cosmopolitan Norms? Rethinking Citizenship in Volatile Times, in: Thomas Faist / Peter Kivisto (Hrsg.), Dual Citizenship in Global Perspective: From Unitary to Multiple Citizenship, Houndmills / UK - New York, S. 247-271.

Benjamin, Thomas (2009): The Atlantic World: Europeans, Africans, Indians and Their Shared History, 1400-1900, Cambridge.

Berger, Johannes (2006): Die Einheit der Moderne, in: Thomas Schwinn (Hrsg.), Die Vielfalt und Einheit der Moderne. Kultur- und strukturvergleichende Analysen, Wiesbaden, S. 201-225.

Berry, Craig (2008): Liberal Political Theorists, the Cosmopolitan Tradition and Globalisation Discourse, in: In-Spire: Journal of Law, Politics and Societies 3, S. 15-32. 
Bonß, Wolfgang / Christoph Lau (Hrsg.) (2010): Macht und Herrschaft in der reflexiven Moderne, Weilerswist.

Boon, Vivienne / Fine, Robert (Hrsg.) (2007): Special Issue on Cosmopolitanism: Between Past and Future, in: European Journal of Social Theory 10 / 1.

Braeckman, Antoon (2008): Reflexive Modernization and the End of the Nation State: On the Eclipse of the Political in Ulrich Beck's Cosmopolitanism, in: Ethical Perspectives 15, S. 343-367.

Brassett, James / Will Smith (2007): Deliberation and Global Governance: Liberal, Cosmopolitan and Critical Perspectives, Garnet Working Paper: No 25/07, October 2007.

Bray, Daniel (2009): Pragmatic Cosmopolitanism: A Deweyan Approach to Democracy beyond the Nation-State, in: Millennium - Journal of International Studies 37, S. 683-719.

Breckenridge, Carol A. / Sheldon Pollock / Homi K. Bhabha / Dipesh Chakrabarty (Hrsg.) (2002): Cosmopolitanism, Durham.

Brock, Gillian / Brighouse, Harry (Hrsg.) (2005): The Political Philosophy of Cosmopolitanism, Cambridge.

Brown, Garrett Wallace (2008): Moving from Cosmopolitan Legal Theory to Legal Practice: Models of Cosmopolitan Law, in: Legal Studies 28, S. 430-451.

Burawoy, Michael (2005): For Public Sociology, in: Soziale Welt 56, S. 347-374.

Calcutt, Lyn / Ian Woodward / Zlatko Skrbis (2009): Conceptualising Otherness: An Exploration of the Cosmopolitan Schema, in: Journal of Sociology 45, S. 169-186.

Calhoun, Craig (2007 a): Cosmopolitanism and Belonging, London.

Calhoun, Craig (2007 b): Nations Matter: Culture, History, and the Cosmopolitan Dream, London - New York.

Calhoun, Craig (2010): Beck, Asia and Second Modernity, in: British Journal of Sociology $61 / 3$, S. 597-619.

Chang, Kyung-Sup (1999): Compressed Modernity and Its Discontents: South Korean Society in Transition, in: Economy and Society 28, S. 30-55.

Chang, Kyung-Sup (2010 a): The Second Modern Condition? Compressed Modernity as Internalized Reflexive Cosmopolitization, in: British Journal of Sociology 61 / 3, S. 444-446.

Chang, Kyung-Sup (2010 b): East Asia's Condensed Transition to Second Modernity, in: Soziale Welt 61/3, S. 319-328.

Cheah, Pheng (2006): Inhuman Conditions: On Cosmopolitanism and Human Rights, Cambridge / MA London.

Conrad, Sebastian / Shalini Randeria (Hrsg.) (2002): Jenseits des Eurozentrismus. Postkoloniale Perspektiven in den Geschichts- und Kulturwissenschaften, Frankfurt / Main.

Constellations: An International Journal of Critical and Democratic Theory (2003) 10 / 4: Cosmopolitanism as Critique.

Daedalus: Journal of the American Academy of Arts and Sciences (2008) 137 / 3: On Cosmopolitanism.

Delanty, Gerard (2009): The Cosmopolitan Imagination: The Renewal of Critical Social Theory, Cambridge.

Delanty, Gerard / Baogang He (2008): Cosmopolitan Perspectives on European and Asian Transnationalism, in: International Sociology 23, S. 323-344.

Delanty, Gerard / Chris Rumford (2005): Rethinking Europe: Social Theory and the Implications of Europeanization, London.

Dobson, Andrew (2006): Thick Cosmopolitanism, in: Political Studies 54, S. 165-184.

Drezner, Daniel W. (2007): All Politics is Global: Explaining International Regulatory Regimes, Princeton. 
Edwards, Kate (2008): For a Geohistorical Cosmopolitanism: Postcolonial State Strategies, Cosmopolitan Communities, and the Production of the 'British', 'Overseas', 'Non-Resident', and 'Global' Indian, in: Environment and Planning D: Society and Space 26, S. 444-463.

Eisenstadt, Shmuel N. (2000 a): Die Vielfalt der Moderne, Weilerswist.

Eisenstadt, Shmuel N. (2000 b): Multiple Modernities, in: Daedalus 129, S. 1-29.

Eisenstadt, Shmuel N. (2006): Die großen Revolutionen und die Kulturen der Moderne, Wiesbaden.

Eriksen, Erik Oddvar (2009): The EU: A Cosmopolitan Vanguard, in: Global Jurist 9, S. 1-23.

Esping-Andersen, Gøsta (1990): The Three Worlds of Welfare Capitalism, Cambridge.

Ewald, François (1993): Der Vorsorgestaat, Frankfurt / Main.

Featherstone, Mike / Heikki Patomäki / John Tomlinson / Couze Venn (Hrsg.) (2002): Special Issue on Cosmopolis: Theory, Culture \& Society 19/1-2.

Fine, Robert (2007): Cosmopolitanism, London - New York.

Fukuyama, Francis (1992): Das Ende der Geschichte. Wo stehen wir?, München.

Garsten, Christina (2003): The Cosmopolitan Organization: An Essay on Corporate Accountability, in: Global Networks 3, S. 355-370.

Giddens, Anthony (1991): Modernity and Self-Identity: Self and Society in the Late Modern Age, Cambridge.

Giddens, Anthony (1996): Konsequenzen der Moderne, Frankfurt / Main.

Giddens, Anthony (1997): Jenseits von Links und Rechts. Die Zukunft radikaler Demokratie, Frankfurt / Main.

Gilroy, Paul (1993): The Black Atlantic: Modernity and Double-Consciousness, Cambridge / MA.

Gilroy, Paul (2008): The Black Atlantic as a Counterculture of Modernity, in: Sanjeev Khagram / Peggy Levitt (Hrsg.), The Transnational Studies Reader: Intersections and Innovations, London, S. 203-216.

Gilroy, Paul (2010): Planetary and Cosmopolitics, in: British Journal of Sociology 61 / 3, S. 620-626.

Glick Schiller, Nina (2009): A Global Perspective on Migration and Development, in: Social Analysis 53, S. 14-37.

Grande, Edgar (2004): Vom Nationalstaat zum transnationalen Politikregime - Staatliche Steuerungsfähigkeit im Zeitalter der Globalisierung, in: Ulrich Beck / Christoph Lau (Hrsg.), Entgrenzung und Entscheidung. Was ist neu an der Theorie reflexiver Modernisierung?, Frankfurt / Main, S. 384-401.

Grande, Edgar (2006): Cosmopolitan Political Science, in: British Journal of Sociology 57, S. 87-111.

Grande, Edgar (2008): Reflexive Modernisierung des Staates, in: der moderne staat. Zeitschrift für Public Policy, Recht und Management 1, S. 7-28.

Grande, Edgar / Markus König / Patrick Pfister / Paul Sterzel (2007): Globalization, Political Transnationalization, and the Future of the Nation-State - A Comparison of Transnational Policy Regimes, in: Stefan A. Schirm (Hrsg.), Globalization. State of the Art and Perspectives, London, S. 98-121.

Grande, Edgar / Louis W. Pauly (Hrsg.) (2005): Complex Sovereignty: Reconstituting Political Authority in the Twenty-first Century, Toronto.

Han, Sang-Jin / Young-Hee Shim (2010): Redefining Second Modernity for East-Asia: A Critical Assessment, in: British Journal of Sociology 61 / 3, S. 465-488.

Hannerz, Ulf (2004): Cosmopolitanism, in: David Nugent / Joan Vincent (Hrsg.), A Companion to the Anthropology of Politics, Oxford, S. 69-85.

Held, David (2007): Soziale Demokratie im globalen Zeitalter, Frankfurt / Main.

Held, David (2010): Cosmopolitanism: Ideals, Realities \& Deficits, Cambridge / UK - Malden / MA.

Held, David / Anthony McGrew / David Goldblatt / Jonathan Perraton (1999): Global Transformations: Politics, Economics and Culture, Cambridge. 
Ishida, Mitsunori et al. (2010): The Individualization of Relationships in Japan, in: Soziale Welt 61 / 3, S. 217-235.

Inglis, David (2009): Cosmopolitan Sociology and the Classical Canon: Ferdinand Tönnies and the Emergence of Global Gesellschaft, in: British Journal of Sociology 60, S. 813-832.

Jessop, Bob (2002): The Future of the Capitalist State, Cambridge.

Katzenstein, Peter J. (2005): A World of Regions: Asia and Europe in the American Imperium, Ithaca.

Kendall, Gavin / Ian Woodward / Zlatko Skrbis (2009): The Sociology of Cosmopolitanism: Globalization, Identity, Culture and Government, London.

Khagram, Sanjeev / Peggy Levitt (Hrsg.) (2008): The Transnational Studies Reader: Intersections and Innovations, London.

Kriesi, Hanspeter / Edgar Grande / Martin Dolezal / Marc Helbling / Dominic Höglinger / Swen Hutter / Bruno Wüest (2010): Restructuring Political Conflict in Western Europe, unveröffentlichtes Manuskript.

Kriesi, Hanspeter / Edgar Grande / Romain Lachat / Martin Dolezal / Simon Bornschier / Timotheos Frey (2008): West European Politics in the Age of Globalization, Cambridge.

Kurasawa, Fuyuki (2004): A Cosmopolitanism from Below: Alternative Globalization and the Creation of a Solidarity without Bounds, in: Archives of European Sociology 45, S. 233-255.

Kurasawa, Fuyuki (2007): The Work of Global Justice: Human Rights as Practices, Cambridge.

Levy, Daniel (2010): Recursive Cosmopolitization: Argentina and the Global Human Rights Regime, in: British Journal of Sociology 61 / 3, S. 579-596.

Levy, Daniel / Michael Heinlein / Lars Breuer (2010): Reflexive Particularism and Cosmopolitanization: The Reconfiguration of the National, in: Global Networks (im Erscheinen).

Levy, Daniel / Natan Sznaider (2007): Erinnerung im globalen Zeitalter. Der Holocaust, Frankfurt / Main.

Levy, Daniel / Natan Sznaider (2010): Human Rights and Memory, University Park / PA.

Luhmann, Niklas (1984): Soziale Systeme. Grundriß einer allgemeinen Theorie, Frankfurt / Main.

Maharaj, Sarat (2010): 'Small Change of the Universal': Beyond Modernity?, in: British Journal of Sociology $61 / 3$, S. 565-578.

Mau, Steffen / Jan Mewes / Ann Zimmermann (2008): Cosmopolitan Attitudes through Transnational Social Practices, in: Global Networks 8, S. 1-24.

Meckled-Garcia, Saladin (2008): On the Very Idea of Cosmopolitan Justice: Constructivism and International Agency, in: The Journal of Political Philosophy 16, S. 245-271.

Mendieta, Eduardo (2009): From Imperial to Dialogical Cosmopolitanism, in: Ethics \& Global Politics 2, S. 241-258.

Meyer, John (2005): Weltkultur. Wie die westlichen Prinzipien die Welt durchdringen, Frankfurt / Main.

Nederveen Pieterse, Jan (2006): Emancipatory Cosmopolitanism: Towards an Agenda, in: Development and Change 37, S. 1247-1257.

Nowicka, Magdalena / Maria Rovisco (Hrsg.) (2009): Cosmopolitanism in Practice, Farnham - Burlington.

Osterhammel, Jürgen (2009): Die Verwandlung der Welt. Die Geschichte des 19. Jahrhunderts, München.

Pichler, Florian (2008): How Real is Cosmopolitanism in Europe?, in: Sociology 42 / 6, S. 1107-1126.

Poferl, Angelika / Natan Sznaider (Hrsg.) (2004): Ulrich Becks kosmopolitisches Projekt. Auf dem Weg in eine andere Soziologie, Baden-Baden.

Pollock, Sheldon / Homi K. Bhabha / Carol A. Breckenridge / Dipesh Chakrabarty (2000): Cosmopolitanism, in: Public Culture 12, S. 577-589.

Pries, Ludger (Hrsg.) (2008 a): Rethinking Transnationalism: The Meso-link of Organizations, Abingdon / Oxon - New York. 
Pries, Ludger (2008 b): Die Transnationalisierung der sozialen Welt. Sozialräume jenseits von Nationalgesellschaften, Frankfurt / Main.

Randeria, Shalini (1999): Jenseits von Soziologie und soziokultureller Anthropologie. Zur Ortsbestimmung der nichtwestlichen Welt in einer zukünftigen Sozialtheorie, in: Soziale Welt 50, S. 373-382.

Randeria, Shalini (2004): Verwobene Moderne. Zivilgesellschaft, Kastenbildung und nicht-staatliches Familienrecht im (post)kolonialen Indien, in: Shalini Randeria / Martin Fuchs / Antje Linkenbach (Hrsg.), Konfigurationen der Moderne. Diskurse zu Indien (Soziale Welt: Sonderband 15), BadenBaden, S. 155-178.

Rapport, Nigel (2007): An Outline for Cosmopolitan Study: Reclaiming the Human through Introspection, in: Current Anthropology 48, S. 257-283.

Sassen, Saskia (1991): The Global City, Princeton.

Sassen, Saskia (1996): Metropolen des Weltmarkts. Die neue Rolle der Global Cities, Frankfurt / Main.

Sassen, Saskia (2008): Das Paradox des Nationalen. Territorium, Autorität und Rechte im globalen Zeitalter, Frankfurt / Main.

Shim, Young-Hee / Sang-Jin Han (2010): "Family-Oriented Individualization" and Second Modernity: An Analysis of Transnational Marriages in Korea, in: Soziale Welt 61 / 3, S. 237-255.

Schwinn, Thomas (Hrsg.) (2006): Die Vielfalt und Einheit der Moderne. Kultur- und strukturvergleichende Analysen, Wiesbaden.

Slaughter, Steven (2009): Reconsidering Institutional Cosmopolitanism: Global Poverty and the Importance of the State in International Political Theory, in: Global Change, Peace \& Security 21, S. 37-52.

Sznaider, Natan (2010): Rewriting the Persian Letters, in: British Journal of Sociology 61 / 3, S. 627-633.

Sørensen, Georg (2004): The Transformation of the State: Beyond the Myth of Retreat, Basingstoke.

Stichweh, Rudolf (2000): Die Weltgesellschaft. Soziologische Analysen, Frankfurt / Main.

The Hedgehog Review: Critical Reflections on Contemporary Culture (2009) 11 / 3: The Cosmopolitan Predicament.

Therborn, Göran (1995): Routes to / through Modernity, in: Mike Featherstone / Scott Lash / Roland Robertson (Hrsg.), Global Modernities, London - Thousand Oaks - New Delhi, S. 124-139.

Tsing, Anna (2009): Supply Chains and the Human Condition, in: Rethinking Marxism 21, S. 148-176.

Turner, Bryan S. (2010): Reflexive Traditionalism and Emergent Cosmopolitanism: Some Reflections on the Religious Imagination, in: Soziale Welt 61 / 3, S. 313-318.

Tyfield, David / Urry, John (2010): Cosmopolitan China?, in: Soziale Welt 61 / 3, S. 277-293.

Vertovec, Steven (2009): Transnationalism, London - New York.

Vertovec, Steven / Cohen, Robin (Hrsg.) (2002): Conceiving Cosmopolitanism: Theory, Context and Practice, Oxford.

Werbner, Pnina (Hrsg.) (2008): Anthropology and the New Cosmopolitanism: Rooted, Feminist and Vernacular Perspectives, Oxford / UK - New York.

Weiß, Anja (2010): Vergleiche jenseits des Nationalstaats. Methodologischer Kosmopolitismus in der soziologischen Forschung über hochqualifizierte Migration, in: Soziale Welt 61 / 3, S. 295-311.

Wilkinson, Iain (2010): Risk, Vulnerability and Everyday Life, Abingdon / Oxon - New York.

Wimmer, Andreas / Nina Glick Schiller (2002): Methodological Nationalism and Beyond: Nation-State Building, Migration and the Social Sciences, in: Global Networks 2, S. 301-334.

Yan, Yunxiang (2010): The Chinese Path to Individualization, in: British Journal of Sociology 61 / 3, S. 489-512.

Young, Robert (2001): Postcolonialism: An Historical Introduction, London.

Ypi, Lea L. (2008): Statist Cosmopolitanism, in: Journal of Political Philosophy 16, S. 48-71. 
Zhang, Joy Yueyue (2010): The Cosmopolitization of Science: Experience from Chinese Stem Cell Scientists, in: Soziale Welt 61 / 3, S. 257-276.

Zürn, Michael (2001): Politik in der postnationalen Konstellation. Über das Elend des methodologischen Nationalismus, in: Christine Landfried (Hrsg.), Politik in einer entgrenzten Welt, Köln, S. 181-203.

Prof. Dr. Ulrich Beck Institut für Soziologie Ludwig-Maximilians-Universität München Konradstr. 6 80801 München u.beck@lmu.de

Prof. Dr. Edgar Grande Geschwister-Scholl-Institut für Politikwissenschaft Ludwig-Maximilians-Universität München Oettingenstr. 67 80538 München grande@1rz.uni-muenchen.de 